\title{
ÜBER DEN ABSOLUTBETRAG STETIGER LINEARER OPERATOREN UND SEINE ANWENDUNG AUF ERGODISCHE ZERLEGUNGEN
}

\author{
ULRICH KRENGEL ${ }^{1}$
}

\section{Einleitung.}

In der Ergodentheorie untersucht man Operatoren $T$ in reellen oder komplexen Banachräumen $X$, in denen gewöhnlich ein positiver Kegel $X^{+}$ausgezeichnet und den Elementen $f \in X$ ein Absolutbetrag $|f| \in X^{+}$ zugeordnet ist, z. B. in $X=L_{p}, 1 \leqq p \leqq \infty$. Viele Resultate sind zunächst für den Spezialfall positiver Operatoren bewiesen worden, $d$. h. für Operatoren, die den positiven Kegel in sich abbilden. Wir nennen hier: 1) den individuellen Ergodensatz (vgl. Dunford-Schwartz [7], [8], ChaconOrnstein [6]) und 2) den Satz über ergodische Zerlegungen (Jacobs [10]). Beide Resultate gestatten auch eine Formulierung für i.a. nicht positive Operatoren. Für den Beweis derartiger Verallgemeinerungen haben Dunford $u$. Schwartz [8] einen Weg gewiesen: Man versucht, den allgemeinen Fall auf den positiven zu reduzieren, indem dem Operator $T$ ein positiver Operator $|T|$, sein Absolutbetrag, zugeordnet wird, der mit $T$ so eng zusammenhängt, daß sich die zu beweisenden Aussagen von $|T|$ auf $T$ übertragen.

Mit dieser Methode bewiesen Dunford u. Schwartz die FastüberallKonvergenz von $(1 / n) \sum_{k=1}^{n-1} f T^{k}$ für $f \in L_{1}$ und beliebige Operatoren $T$ in $L_{1}$, die außer der $L_{1}$-Norm auch die $L_{\infty}$-Norm kontrahieren. Chacon $u$. Krengel [5] zeigten, daß sich $|T|$ auch konstruieren läßt, wenn $T$ nur die $L_{1}$-Norm kontrahiert, und Chacon [4] hat damit die bisher allgemeinste Form des individuellen Ergodensatzes bewiesen. Diese umfaßt auch die Version von Chacon-Ornstein [6] des Ergodensatzes sowie deren in Abschnitt 9 beschriebene Ausdehnung auf beliebige Operatoren mit Hilfe von $|T|$.

Gegenstand der vorliegenden Arbeit ist eine systematische Untersuchung der Zuordnung $T \rightarrow|T|$ für lineare stetige Operatoren $T$ zwischen komplexen Banachräumen mit positivem Kegel (sog. (Ko,B)-Räumen).

Eingegangen am 4. Oktober, 1963.

1 Die vorliegenden Untersuchungen wurden als Dissertation bei der Universität Göttingen eingereicht. (Satz 3.3 ist jedoch später zugefügt worden.) 
Die früheren Resultate von Dunford-Schwartz und Chacon-Krengel ergeben sich als Spezialfall (vgl. Sätze 3.2 und 4.1).

Dabei wird der Ưbergang zu reellen Banachräumen i.a. nicht eigens erwähnt. Im reellen Fall ergeben sich Berührungspunkte mit Arbeiten von F. Riesz [16] und L. Kantorovitch [14] über Verbandsaussagen in einigen Räumen von reellen Operatoren. (Auf Letztere wurde ich erst nach Abschluß dieser Arbeit aufmerksam. Sie behandelt vor allem in Ordnungstopologien stetige Operatoren, enthält aber bereits das wichtige Corollar 4.1.)

Als Anwendung erhalten wir in Abschnitt 10 die ergodische Zerlegung stetiger Operatoren im reellen oder komplexen $L_{1}$ ohne Positivitätsforderung, teils durch Reduktion auf die oben unter 2) erwähnte Aussage, teils durch direkte Einführung von $|T|$ in die für 2) gegebenen Beweise.

In Abschnitt 1 fassen wir die wichtigsten natürlichen Forderungen an $X$ und $Y$ zum Begriff des $(K o, B)$-Raumes (im reellen Fall: des $(B, V)$ Raumes $=$ Banachverbandes mit $\||f|\|=\|f\|)$ zusammen und zählen Beispiele auf. Abschnitt 2 stellt nur den Zusammenhang mit den Verbandsaussagen her: Die stetigen linearen Operatoren zwischen $(B, V)$-Räumen $X$ und $Y$ bilden einen bedingt vollständigen Banachverband, wenn $Y$ bedingt vollständig ist - ein bis auf die Stetigkeitsbedingung bekanntes Resultat. Dieser Satz gibt Anlaß zur Definition der Majorisierbarkeit von Operatoren. Aus ihr folgt für Räume $Y$, deren Realteil ein bedingt vollständiger Banachverband ist, die Existenz von $|T|$, nicht dagegen z. B. für $Y=C(\Omega)$.

Im dritten Abschnitt zeigen wir die Äquivalenz dreier Darstellungen von $|T|$. Die nützlichste erste ähnelt der Konstruktion bei ChaconKrengel, ist aber nicht auf die spezielle Struktur von $L_{1}$ angewiesen, im reellen Fall ist sie bei Kantorovitch angedeutet. Die zweite hat die dem Ansatz von Dunford-Schwartz entsprechende Gestalt (Satz 3.2), die dritte zeigt, daß sich der Absolutbetrag $|T|$ wie der von $f \in X$ bilden läßt. (Satz 3.3).

Im vierten Abschnitt werden Majorisierbarkeits-Kriterien gegeben: Es zeigt sich, daß es hinreichend ist, wenn $X$ die Eigenschaft $(L)$ hat, d. h. für $f, g \in X^{+}$stets $\|f+g\|=\|f\|+\|g\|$ gilt, oder aber wenn $Y$ eine Einheit 1 besitzt, d. h. ein Element $1 \geqq 0$ mit den Eigenschaften: a) $\|1\|=1$ und b) für $h \in Y$ mit $\|h\| \leqq 1$ ist $|h| \leqq 1$. Hier zeigt sich, daß sich auch die Theorie der Totalvariation komplexer Maße einordnet, die wir dazu als lineare Funktionale auf einem passenden $L_{1}$ auffassen.

Im fünften Abschnitt wird gezeigt, daß Operatoren in $L_{2}=X=Y$ i. a. nicht majorisierbar sind: Im reellen $L_{2}$ bilden die stetigen Operatoren 
genau dann einen Verband, wenn der $L_{2}$ endlich-dimensional ist. Im sechsten Abschnitt schliessen wir von der Majorisierbarkeit von $T$ auf die des dualen Operators $T^{*}$ und beweisen in einigen Fällen $\left|T^{*}\right|=|T|^{*}$.

Von da an legen wir zugrunde, daß $X$ die Eigenschaft $(L)$ hat. Wir zeigen, daß für vollstetige Operatoren $T$ auch $|T|$ vollstetig ist. Weiter geben wir eine Klasse von Operatoren $T$ an, für die sich $|T|$ finit bestimmen läßt, und die im Raum aller stetigen Operatoren von $X$ nach $Y$ dicht in der starken Operatortopologie ist. Allerdings gilt für den Übergang $T \rightarrow|T|$ nur monotone Stetigkeit in dieser Topologie.

Ich danke Herrn Prof. K. Jacobs vielmals für sein Interesse an der Arbeit und äußerst wertvolle Anregungen.

\section{Definitionen und Hilfsmittel.}

$H$ sei eine halbgeordnete Menge, $\leqq$ bezeichne die Halbordnung. Existiert zu je zwei Elementen $f, g \in H$ eine kleinste obere Schranke $f v g$ und eine größte untere Schranke $f \wedge g$, so heißt $H$ ein Verband. $H$ heißt ein bedingt vollständiger Verband, wenn zu jeder nach oben beschränkten Teilmenge $H_{0} \subseteq H$ eine kleinste obere Schranke (sup) und zu jeder nach unten beschränkten Teilmenge $H_{0} \subseteq H$ eine größte untere Schranke (inf) existiert. (Vgl. Jacobs [9, S. 155]).

Ist ein Verband $H$ zugleich ein reeller linearer Raum und die Halbordnung gegen Translation und Multiplikation mit positiven Zahlen invariant, so heißt $H$ ein Vektorverband. In diesem sei $f^{+}=f \vee 0, f^{-}=$ $(-f) \vee 0$ und $|f|=f \vee(-f)$. Es gilt $f=f^{+}-f^{-},|f|=f^{+}+f^{-}, f^{+} \wedge f^{-}=0$, $f+g=(f \vee g)+(f \wedge g)$ und die Gleichungen $a \wedge b=0$ und $a \wedge c=0$ implizieren zusammen $a \wedge(b+c)=0$ (vgl. Birkhoff $[1, \mathrm{~S} .219-220])$.

Für beliebige halbgeordnete lineare Räume $X$ sei $X^{+}=\{f \in X: f \geqq 0\}$.

Ist ein Vektorverband $H$ zugleich ein reeller Banachraum, gilt $\|f\| \leqq\|g\|$ für $f, g \in H^{+}$und $f \leqq g$, und gilt für gegen $f$ bzw. $g$ normkonvergente Folgen $\left\{f_{n}\right\}$ und $\left\{g_{n}\right\}$ mit $f_{n} \leqq g_{n}$ stets $f \leqq g$, so heißt $H$ Banachverband. Alle Verbandsoperationen sind dann stetig. Wir nehmen durchgehend symmetrische Normen: $\|f\|=\||f|\|$ an und nennen Banachverbände mit dieser Eigenschaft $(B, V)$-Räume.

Ein komplexer Banachraum $X$ heiße $(K o, B)$-Raum oder auch komplexe Hülle des Banachverbandes $H=\operatorname{Re}(X)$, wenn er folgende Eigenschaften hat:

(1.1) $\operatorname{Re}(X)=H \cong X$.

(1.2) Jedes $f \in X$ ist eindeutig darstellbar als Summe $f=\operatorname{Re}(f)+$ $i \operatorname{Im}(f) \operatorname{mit} \operatorname{Re}(f) \in H, \operatorname{Im}(f) \in H$.

(1.3) Jedem Element $f \in X$ ist ein Element $|f| \in H^{+}$so zugeordnet, 
daß $|f|$ der im Banachverband $H$ definierte Absolutbetrag ist, wenn $f \in H$.

Es gelte ferner:

(1.4) $\|f\|=\||f|\|, f \in X$.

(1.5) Die Abbildungen $f \rightarrow|f|$ und $f \rightarrow \operatorname{Re}(f)$ sind normstetig.

(1.6) Es ist $|f|=\sup _{|a|=1} \operatorname{Re}(a f)$.

(1.7) Der Raum $\operatorname{Tr}(X)=\left\{f \in X: f=\sum_{i=1}^{n} a_{i} f_{i}, a_{i}\right.$ komplex, $f_{i} \in H^{+}$und $\left.f_{i} \wedge f_{k}=0, i \neq k\right\}$ ist normdicht in $X$.

Aus (1.6) folgt für $f \in \operatorname{Tr}(X)$ die Formel $|f|=\sum_{i=1}^{n}\left|a_{i}\right| f_{i}$.

$(B, V)$-Räume besitzen alle wichtigen Struktureigenschaften der $(K o, B)$-Räume in reeller Gestalt und daher formulieren wir Definitionen und Sätze meist nur für $(K o, B)$-Räume, obwohl sich alles auf $(B, V)$ Räume überträgt. Wir sagen, die komplexe Hülle $X$ von $H$ habe die Eigenschaft:

$(b v)$ : wenn $H$ ein bedingt vollständiger Verband ist.

$(L)$ : wenn $\|f+g\|=\|f\|+\|g\|$ für $f, g \in H^{+}=X^{+}$gilt.

$(M)$ : wenn $\|f \vee g\|=\max \{\|f\|,\|g\|\}$ für $f, g \in H^{+}$gilt.

(1): wenn $X^{+}$eine Einheit 1 enthält, d. h. ein Element $1 \in X^{+}$mit $\|1\|=1$, für das $|f| \leqq 1$ für diejenigen $f \in X$ ist, für die $\|f\| \leqq 1$ ist.

$(m K)$ : wenn in $H$ der Satz von der monotonen Konvergenz gilt.

Erläuterung zu $(m K)$ : Ist $I$ eine aufsteigend gefilterte Indexmenge, d. h. eine Moore-Smith-Folge mit der Halbordnung $\leqq$, und ist jedem $i \in I$ ein $f_{i} \in H$ zugeordnet, so nennen wir das System $\left\{f_{i}, i \in I\right\}$ monoton wachsend, wenn für $i_{1} \leqq i_{2}$ stets $f_{i_{1}} \leqq f_{i_{2}}$ ist. Wir sagen, daß in $H$ der Satz von der monotonen Konvergenz gilt, wenn zu jedem normbeschränkten monoton wachsenden System $\left\{f_{i}, i \in I\right\}$ ein $f \in H$ existiert, das die Eigenschaft hat: $\mathrm{Zu} \varepsilon>0$ gibt es ein $i_{\varepsilon} \in I$ derart, daß $\left\|f-f_{i}\right\|<\varepsilon$ für $i \geqq i_{\varepsilon^{\prime}}$. Dann folgt $f \geqq f_{i}$ für $i \in I$ und $\|f\| \leqq \sup \left\{\left\|f_{i}\right\|\right\}$.

Aus $(m K)$ folgt $(b v)$ : Ist etwa $f_{k} \leqq h$ für $k \in K \neq 0$ und $k_{0} \in K$, so ist $f=\sup \left\{f_{k}\right\}$ genau dann, wenn $f=\sup \left\{f_{k} \vee f_{k_{0}}\right\}$ ist, und nach einer Translation können wir $0 \leqq f_{k} \leqq h, k \in K$, annehmen. I sei das aufsteigend gefilterte System der endlichen Teilmengen $i=\left\{k_{1}, \ldots, k_{n}\right\}$ von $K, i_{1} \leqq i_{2}$ ist in $I$ durch $i_{1} \subseteq i_{2}$ definiert. Wir bilden das monoton wachsende und durch $\|h\|$ normbeschränkte System

$$
g_{i}=f_{k_{1}} \vee \ldots \vee f_{k_{n}} \text {. }
$$

Ist $f=\lim _{i \in I} g_{i}$, dann ist $f \geqq g_{i}$ für alle $i$, also $\geqq f_{k}, k \in K$. Umgekehrt zeigt man durch Auswahl einer konvergenten Folge von $g_{i}$ daß $f \leqq h$, und, da $h$ eine beliebige Schranke war, folgt $f=\sup \left\{f_{k}\right\}$.

Um $(m K)$ nachzuweisen, braucht man in $\left\{f_{i}, i \in I\right\}$ stets nur solche $i \in I \mathrm{zu}$ betrachten, die größer als ein festes $i_{0} \in I$ sind und nach einer 
Translation kann man dann stets $f_{i} \geqq 0$ annehmen. So zeigt man, daß $(m K)$ und also auch $(b v)$ aus $(L)$ folgt.

Einen $(K o, B)$-Raum mit den Eigenschaften $(1)$ und $(b v)$ nennen wir $(K o, B, 1, b v)$-Raum und entsprechend sind andere Bezeichnungen zu verstehen, etwa $(K o, B, L)$-Raum oder $(B, V, m K)$-Raum. Dabei verwenden wir statt der Ausdrücke $(B, V, L)$-Raum und $(B, V, M)$-Raum in Anlehnung an Kakutani [13] auch die Bezeichnungen $(L)$-Raum und $(M)$ Raum.

Beispiel 1. Der Raum $(\Omega, B, m)$ sei ein Maßraum und $L_{p}=L_{p}(m)$ der komplexe $L_{p}(\Omega, B, m), 1 \leqq p \leqq \infty$. Es ist:

(1a) $L_{1}$ ein $(K o, B, L)$-Raum;

(1b) $\quad L_{p}$ ein $(K o, B, m K)$-Raum, $1 \leqq p<\infty$;

(1c) $L_{\infty}$ ein $(K o, B, 1, M)$-Raum und für $\sigma$-endliches $m$ gilt außerdem $(b v)$.

In dieser Arbeit sind Gleichungen und Ungleichungen zwischen Funktionen aus $L_{p}, 1 \leqq p \leqq \infty$, stets als fastüberall geltend zu verstehen.

Beweishinweise: Bezüglich der (in $L_{p}$ trivialen) Verbandsstruktur und der Banachraumstruktur verweise ich - zugleich für die folgenden Beispiele auf Dunford-Schwartz [7]. Die Eigenschaften $(L)$ in (la) und $(M)$ in (1c) folgen aus den Definitionen. Einheit 1 in $L_{\infty}$ ist die Konstante 1. Bezüglich $(m K)$ in (lb) siehe Bourbaki [2, Chap. IV., 3.6]. Ist $m \sigma$-endlich, so folgt $(b v)$ in (1c) aus Dunford-Schwartz [7, Satz IV, 8.23]. (Ein Gegenbeispiel, wenn $m$ nicht $\sigma$-endlich ist: $\Omega=[0,1], B=$ Borelsche Mengen, $m$ : Jeder Punkt erhält die Masse 1. Sei $f_{x}(\omega)=\chi_{\{x\}}(\omega)$. Ist $A$ nicht Borel-meßbar, so existiert nicht $\sup _{x \in A} f_{x}$ in $L_{\infty}$.) Ist eine Folge $f_{k}$ im reellen $L_{p}, 1 \leqq p \leqq \infty$, durch $h \in L_{p}$ beschränkt: $h \geqq f_{k}$, so führt Supremumsbildung nicht aus dem Bereich der meßbaren Funktionen heraus und es ist $\sup f_{k} \in L_{p}$, wobei $\sup f_{k}$ punktweise gebildet wird - unabhängig von den Repräsentanten, da nur abzählbar viele $f_{k}$ berücksichtigt werden. So beweist man (1.6), indem $\sup _{|a|=1} \operatorname{Re}(a f)$ nur über solche $a$ erstreckt wird, die einen rationalen Realteil haben. Da solche $a$ dicht im Einheitskreis liegen, erhält man so bereits das volle Supremum. (1.7) gilt, da sogar die Treppenfunktionen normdicht liegen. (1.5) folgt aus

$$
|| f|-| g|| \leqq|f-g| \text { und }|\operatorname{Re}(f)-\operatorname{Re}(g)| \leqq|f-g| \text {. }
$$

Beispiel 2. Der Raum $K(B)$ der komplexen Ladungsverteilungen, d. h. der verallgemeinerten Maße auf $(\Omega, B)$ ist ein $(K o, B, L)$-Raum. (Jacobs [9, S. 165]); denn zu $h \in K(B)$ kann man die Totalvariation $|h| \in K(B)$ bestimmen. Setzt man ||$h||=|h|(\Omega)$ und $|h|=m$, so hat $h$ nach dem Satz 
von Radon-Nikodym die Darstellung $h(E)=\int_{E} f d m$, und diese benutzt man, um den Beweis auf Beispiel 1 zu reduzieren.

Beispiel 3. Ist $\Omega$ eine beliebige Punktmenge, so ist $M(\Omega)$, der Raum der beschränkten komplexen Funktionen auf $\Omega$, ein $(K o, B, 1, b v, M)$ Raum mit $\|f\|=\sup _{\omega \in \Omega}|f(\omega)|$, denn man kann $M(\Omega)$ als speziellen Raum $L_{\infty}$ auffassen und $(b v)$ gilt, da man punktweise das Supremum bilden kann.

BeISPIEL 4. Ist $\Omega$ ein topologischer Raum, so ist der Raum $C(\Omega)$ der reellen stetigen Funktionen auf $\Omega$ ein $(B, V, M, 1)$-Raum, wenn $\|f\|$ wie in Beispiel 3 gebildet ist. Der zugehörige komplexe Raum ist im allgemeinen kein $(K o, B)$-Raum.

Andere Räume werden in dieser Arbeit nicht benötigt. Es lassen sich jedoch noch zahlreiche Beispiele nennen, u. a. der Raum der Funktionen von beschränkter Totalvariation in einem Intervall als weiterer $(K o, B, L)$-Raum und der Raum der komplexen Nullfolgen $\left\{x_{n}\right\}$ mit $\left\|\left\{x_{n}\right\}\right\|=\sup \left|x_{n}\right|$ als $(K o, B, b v, M)$-Raum ohne Einheit (vgl. DunfordSchwartz [7, S. 238]).

Positive Operatoren. Sind $X$ und $Y(K o, B)$-Räume, so nennen wir einen linearen Operator $T$ von $X$ nach $Y$ positiv, in Zeichen $T \geqq 0$, wenn $f T \in Y^{+}$für alle $f \in X^{+}$ist. Eine Halbordnung wird erklärt, indem wir für zwei Operatoren $T_{1}, T_{2}, T_{1} \geqq T_{2}$ statt $\left(T_{1}-T_{2}\right) \geqq 0$ schreiben. $T$ heißt reell, wenn für $f \in \operatorname{Re}(X)$ stets $f T \in \operatorname{Re}(Y)$ ist.

Stetige lineare Operatoren $T$ von $X$ nach $Y$ sind lineare Operatoren mit endlicher Norm:

$$
\|T\|=\sup _{\|f\| \leqq 1}\|f T\| .
$$

Ist $(\Omega, B, m)$ ein Maßraum, $X=L_{p}$ und $Y=L_{p}$, und bedeutet $\|f\|_{q}$ die Norm in $L_{q}$ von $f \in L_{p} \cap L_{q}, 1 \leqq p, q \leqq \infty$, so schreiben wir

$$
\|T\|_{q}=\sup _{\|f\| g \leqq 1}\|f T\|_{q},
$$

$\|\cdot\|$ anstelle von $\|\cdot\|_{1}$ und, wenn nur eine Norm betrachtet wird, auch anstelle von $\|\cdot\|_{p}$.

Sind $X$ und $Y(K o, B)$-Räume und ist ein Operator $T$ auf $X^{+}$definiert, dort additiv und positiv homogen, d. h. gilt (af)T=a(fT) für $a \geqq 0$ und $f \in X^{+}$, ist ferner $f T \in Y^{+}, f \in X^{+}$, und gilt $\|f T\| \leqq K\|f\|$ für eine Konstante $K<\infty$, so läßt sich $T$ zu einem linearen stetigen positiven Operator auf $X$ fortsetzen: Ist $h=h_{1}-h_{2}=h_{1}{ }^{\prime}-h_{2}{ }^{\prime}, h_{k}, h_{k}{ }^{\prime} \in X^{+}$, so ist $h_{1} T-h_{2} T=h_{1}{ }^{\prime} T-h_{2}{ }^{\prime} T$, da $T$ additiv ist, und wir können dies $=h T$ setzen. Ist $f=h_{r}+i h_{i}, h_{r}, h_{i} \in \operatorname{Re}(X)$, so setzen wir $f T=h_{r} T+i\left(h_{i} T\right)$. Dann ist $T$ linear. Es gilt 


$$
\|f T\|=\left\|h_{r}+T-h_{r}-T+i h_{i}+T-i h_{i}-T\right\| \leqq 4 K\|f\| .
$$

$T$ ist also stetig. Es gilt aber sogar

$$
\|T\| \leqq K .
$$

Um dies zu zeigen, bemerken wir, daß aus (1.6) und

$$
\sup \left\{f_{i}\right\}+\sup \left\{g_{i}\right\} \geqq \sup \left\{f_{i}+g_{i}\right\}
$$

folgt, daß für $f, g \in X$ stets

$$
|g+h| \leqq|g|+|h|
$$

gilt. Ist $f=\sum_{i=1}^{n} a_{i} f_{i} \in \operatorname{Tr}(X)$, so ist

und also

$$
\left|\sum_{i=1}^{n} a_{i} f_{i} T\right| \leqq \sum_{i=1}^{n}\left|a_{i} f_{i} T\right|=\sum_{i=1}^{n}\left|a_{i}\right| f_{i} T
$$

$$
\begin{aligned}
\|f T\|=\left\|\sum_{i=1}^{n} a_{i} f_{i} T \mid\right\| & \leqq\left\|\sum_{i=1}^{n}\left|a_{i}\right| f_{i} T\right\| \\
& \leqq\left\|\sum_{i=1}^{n}\left|a_{i}\right| f_{i}\right\| K=\|f\| K .
\end{aligned}
$$

$\operatorname{Da} \operatorname{Tr}(X)$ dicht in $X$ ist, erhalten wir $\|T\| \leqq K$.

Bemerkung. Die Bedingung (1.7) spielt - wie wir sehen - nicht eine ausschlaggebende Rolle und wir verwenden sie nur, um eine geschlossene Darstellung zu erreichen. Später wird sie wesentlich nur noch zum Beweis von Lemma 3.1 benötigt. Im Bildraum $Y$ des Operators ist sie zur Durchführung der Theorie völlig entbehrlich.

\section{Das Supremum beschränkter Mengen von Operatoren.}

Lemma 2.1. Ist $X$ ein Vektorverband und sind

$$
Z_{1}(f)=\left\{f_{11}, \ldots, f_{1 r}\right\} \quad \text { und } \quad Z_{2}(f)=\left\{f_{21}, \ldots, f_{2 s}\right\}
$$

zwei Zerlegungen von $f \in X^{+}$, das heißt n-tupel $\left\{f_{1}, \ldots, f_{n}\right\}$ mit $f_{i} \in X^{+}$und $\sum_{i=1}^{n} f_{i}=f$, so existiert zu diesen eine Verfeinerung, d.h. eine Zerlegung $\left\{g_{11}, \ldots, g_{r s}\right\}$ mit $\sum_{i=1}^{r} g_{i k}=f_{2 k}$ und $\sum_{k=1}^{s} g_{i k}=f_{1 i}$.

Für $s=1$ ist dies trivial, für $s=2$ hat es Kantorovitch [14, S. 220] bewiesen und für beliebiges $s$ folgt es durch Induktion. Die Zerlegungen $Z(f)$ eines Elements $f \in X^{+}$bilden also bezüglich der durch den Begriff der Verfeinerung bestimmten Halbordnung ein gefiltertes System $\mathbb{Z}(f)$.

SaTz 2.1. Der Banachraum $H$ aller stetigen linearen Operatoren von einem $(B, V)$-Raum $X$ in einen $(B, V, b v)$-Raum $Y$ ist ein bedingt vollständiger Verband. 
BEweIs. Es sei $T_{0} \in H, L$ eine nicht leere Indexmenge und $T_{0} \geqq T_{\lambda} \in H$, $\lambda \in L$. Wir konstruieren $\tilde{T}=\sup _{\lambda \in L} T_{\lambda}$. Für $f \in X^{+}$, eine Zerlegung

und $\left(\lambda_{1}, \ldots, \lambda_{k}\right) \subseteq L$ sei

$$
Z(f)=\left\{f_{1}, \ldots, f_{k}\right\}
$$

und

$$
Q\left(Z(f), \lambda_{1}, \ldots, \lambda_{k}\right)=\sum_{i=1}^{k} f_{i} T_{\lambda_{i}} \leqq f T_{0}
$$

$$
f \widetilde{T}=\sup _{\substack{Z(f) \in B(f) \\\left(\lambda_{1}, \ldots, \lambda_{k}\right) \cong L}} Q\left(Z(f), \lambda_{1}, \ldots, \lambda_{k}\right) .
$$

Da $Y$ ein $(B, V, b v)$-Raum ist, ist $f \tilde{T} \in Y$. Aus der Definition folgt, daß $\widetilde{T}$ auf $X^{+}$positiv homogen ist.

Wir zeigen, daß für $f, g \in X^{+}$

$$
(f+g) \tilde{T}=f \widetilde{T}+g \widetilde{T}
$$

gilt: Ist $Z^{\prime}(f)=\left\{f_{1}, \ldots, f_{k}\right\}$ und $Z^{\prime \prime}(g)=\left\{g_{1}, \ldots, g_{j}\right\}$, so bilden wir

Dann ist

$$
Z(f+g)=\left\{f_{1}, \ldots, f_{k}, g_{1}, \ldots, g_{j}\right\}
$$

$$
\begin{aligned}
Q\left(Z^{\prime}(f), \lambda_{1}, \ldots, \lambda_{k}\right)+Q\left(Z^{\prime \prime}(g), \mu_{1}, \ldots, \mu_{j}\right) & =Q\left(Z(f+g), \lambda_{1}, \ldots, \mu_{j}\right) \\
& \leqq(f+g) \tilde{T},
\end{aligned}
$$

und daraus folgt $f \widetilde{T}+g \widetilde{T} \leqq(f+g) \widetilde{T}$. Ist aber

$$
Z(f+g)=\left\{h_{1}, \ldots, h_{k}\right\} \quad \text { und } \quad\left(\lambda_{1}, \ldots, \lambda_{k}\right)
$$

vorgegeben, so bilden wir eine Verfeinerung

$$
\left\{f_{1}, \ldots, f_{k}, g_{1}, \ldots, g_{k}\right\}
$$

von $Z(f+g)$ und $\{f, g\}$. Setzen wir $Z^{\prime}(f)=\left\{f_{1}, \ldots, f_{k}\right\}$ und $Z^{\prime \prime}(g)=$ $\left\{g_{1}, \ldots, g_{k}\right\}$, so ist

$$
\begin{aligned}
Q\left(Z(f+g), \lambda_{1}, \ldots, \lambda_{k}\right) & =Q\left(Z^{\prime}(f), \lambda_{1}, \ldots, \lambda_{k}\right)+Q\left(Z^{\prime \prime}(g), \lambda_{1}, \ldots, \lambda_{k}\right) \\
& \leqq f \tilde{T}+g \tilde{T}
\end{aligned}
$$

und also $(f+g) \widetilde{T} \leqq f \widetilde{T}+g \widetilde{T}$. Da mithin $\widetilde{T}$ auf $X^{+}$additiv ist, können wir es durch $f \tilde{T}=f+\tilde{T}-f-\tilde{T}$ zu einem auf ganz $X$ definierten linearen Operator $\tilde{T}$ fortsetzen.

Dieser Operator $\widetilde{T}$ ist stetig. Um dies zu zeigen, betrachten wir statt $T_{\lambda}$ nun $T_{\lambda}{ }^{\prime}=T_{\lambda}-T_{0}$. Wir erhalten

$$
T_{\lambda}^{\prime} \leqq T^{\prime}=\tilde{T}-T_{0} \leqq 0 .
$$

Für beliebiges festgewähltes $\lambda \in L$ und $f \in X$ ist 


$$
f^{+} T_{\lambda}{ }^{\prime} \leqq f^{+} \widetilde{T}^{\prime} \leqq 0 \quad \text { und } \quad f-T_{\lambda}{ }^{\prime} \leqq f-\widetilde{T}^{\prime} \leqq 0 .
$$

Aus der Monotonie der Norm in $Y$ und $X$ folgt

ferner

$$
\left\|f^{+} \tilde{T}^{\prime}\right\| \leqq\left\|f^{+} T_{\lambda}^{\prime}\right\| \quad \text { und } \quad\left\|f^{-} \tilde{T}^{\prime}\right\| \leqq\left\|f^{-} T_{\lambda}^{\prime}\right\|,
$$

$$
\left\|f^{+}\right\| \leqq\|f\|\|=\| f \| \quad \text { und } \quad\left\|f^{-}\right\| \leqq\||f|\|=\|f\| .
$$

Insgesamt ergibt sich

$$
\left\|f \widetilde{T}^{\prime}\right\| \leqq\left\|f+\widetilde{T}^{\prime}\right\|+\left\|f-\widetilde{T}^{\prime}\right\| \leqq 2\left\|T_{\lambda}{ }^{\prime}\right\|\|f\| .
$$

Aus der Stetigkeit von $\widetilde{T}^{\prime}$ und $T_{0}$ folgt die von $\widetilde{T}$.

Da $T_{\lambda} \leqq \widetilde{T} \leqq T_{0}$ für jedes $\lambda \in L$ gilt, $T_{0}$ aber eine beliebige Schranke der $T_{\lambda}$ war, ist $\widetilde{T}=\sup _{\lambda \in L} T_{\lambda}$.

Bemerkung. Die eben angewandte Methode ist ohne Stetigkeitsüberlegungen schon von Kantorovitch [14, S. 221] zum Beweis eines anderen Resultats entwickelt worden, zugleich von Riesz [16, S. 179], für positive Operatoren, ebenfalls ohne Stetigkeitsbetrachtungen.

Definition 2.1. Ein stetiger linearer Operator von einem $(K o, B)$ Raum, bzw. $(B, V)$-Raum $X$ in einen $(K o, B)$-Raum, bzw. $(B, V)$-Raum $Y$ heißt majorisierbar, wenn für mindestens einen positiven stetigen linearen Operator $\tilde{T}$

$$
f \widetilde{T} \geqq|f T|, \quad f \in X^{+},
$$

gilt. $\tilde{T}$ heißt Majorante von $T$.

Die majorisierbaren Operatoren bilden einen linearen Raum. Ist $X$ ein $(B, V)$-Raum und $Y$ ein $(B, V, b v)$-Raum, so bilden sie nach Satz 2.1 einen Verband. Umgekehrt sind reelle Operatoren, die Differenz zweier positiver Operatoren sind, stets majorisierbar. Beispiel 5 in Abschnitt 4 wird $u$. a. zeigen, daß für einen majorisierbaren Operator $T$ von einem $(B, V)$-Raum $X$ in einen $(B, V)$-Raum $Y$ nicht notwendig $T^{+}=T \vee 0$ existiert, daß also in Satz 2.1 die Bedingung (bv) für $Y$ wesentlich ist.

\section{Die Darstellungen des Absolutbetrages $|\boldsymbol{T}|$ von $\boldsymbol{T}$.}

Ist $T$ ein majorisierbarer Operator von einem $(B, V)$-Raum in einen $(B, V, b v)$-Raum, so können wir Satz 2.1 anwenden und $T \vee(-T)=|T|$ und $T \vee 0=T^{+}$so bilden, daß für $f \in X^{+}$

$$
f|T|=\sup _{0 \leqq f^{\prime} \leqq f}\left\{f^{\prime} T-\left(f-f^{\prime}\right) T\right\}
$$

und 


$$
f^{+}=\sup _{0 \leqq f^{\prime} \leqq f} f^{\prime} T
$$

ist. (Kantorovitch [14, S. 222]). Sei nun $T$ ein majorisierbarer Operator von einem $(K o, B)$-Raum $X$ in einen $(K o, B, b v)$-Raum $Y$ und $T_{0}$ eine Majorante. Zu $f \in X^{+}$und

bilden wir

$$
Z(f)=\left\{f_{1}, \ldots, f_{k}\right\}
$$

$$
\begin{aligned}
& Q^{r+(Z(f), T)}=\sum_{i=1}^{k}\left(\operatorname{Re}\left(f_{i} T\right) \vee 0\right) \leqq f T_{0}, \\
& Q^{r-(Z(f), T)}=\sum_{i=1}^{k}\left(\operatorname{Re}\left(-f_{i} T\right) \vee 0\right) \leqq f T_{0}, \\
& Q^{(\sim)}(Z(f), T)=\sum_{i=1}^{k}\left|f_{i} T\right| \leqq f T_{0} .
\end{aligned}
$$

(*) bezeichne ein beliebiges der Zeichen $(\sim), r+, r-$. Bei Verfeinerung von $Z(f)$ ist $Q^{(*)}(Z(f), T)$ monoton wachsend; denn ist $f_{i}=\sum_{j=1}^{n} f_{i j}$, so ist

ebenso

$$
\operatorname{Re}\left(f_{i} T\right) \vee 0=\left(\sum_{j=1}^{n} \operatorname{Re}\left(f_{i j} T\right)\right) \vee 0 \leqq \sum_{j=1}^{n}\left(\operatorname{Re}\left(f_{i j} T\right) \vee 0\right),
$$

und

$$
\operatorname{Re}\left(-f_{i} T\right) \vee 0 \leqq \sum_{j=1}^{n}\left(\operatorname{Re}\left(-f_{i j} T\right) \vee 0\right)
$$

$$
\left|f_{i} T\right| \leqq \sum_{j=1}^{n}\left|f_{i j} T\right|
$$

Da in $Y^{+}$das Supremum beschränkter Mengen existiert, definieren wir

$$
f T^{(*)}=\sup _{Z(f) \in B(f)} Q^{(*)}(Z(f), T) \leqq f T_{\mathbf{0}} .
$$

$T^{(*)}$ ist auf $Y^{+}$positiv homogen und sogar additiv: Sei $f, g \in X^{+}$. Für vorgegebenes $Z(f)$ und $Z(g)$ ist $Z(f+g)=Z(f) \cup Z(g)$ so fein, daß

$$
Q^{(*)}(Z(f), T)+Q^{(*)}(Z(g), T)=Q^{(*)}(Z(f+g), T) \leqq(f+g) T^{(*)}
$$

gilt. Es folgt $f T^{(*)}+g T^{(*)} \leqq(f+g) T^{(*)}$. Ist $Z(f+g)$ vorgegeben, so verfeinern wir es nach Lemma 2.1 mit $\{f, g\}$ zu $Z^{\prime}(f+g)=Z^{\prime}(f) \cup Z^{\prime}(g)$. Dann ist

$$
\begin{aligned}
Q^{(*)}(Z(f+g), T) & \leqq Q^{(*)}\left(Z^{\prime}(f+g), T\right) \\
& =Q^{(*)}\left(Z^{\prime}(f), T\right)+Q^{(*)}\left(Z^{\prime}(g), T\right) \\
& \leqq f T^{(*)}+g T^{(*)} .
\end{aligned}
$$

Daraus folgt $(f+g) T^{(*)} \leqq f T^{(*)}+g T^{(*)}$ und also

$$
(f+g) T^{(*)}=f T^{*}+g T^{*} .
$$


Für $f \in X^{+}$ist $\left\|f T^{(*)}\right\| \leqq\left\|f T_{0}\right\| \leqq\|f\|\left\|T_{0}\right\|$, und $T^{(*)}$ läßt sich daher zu einem linearen Operator auf $X$ mit $\left\|T^{(*)}\right\| \leqq\left\|T_{0}\right\|$ fortsetzen. Konstruiert man im reellen Fall $T^{(*)}$ ebenso, so gilt:

SaTz 3.1. Ist $T$ ein majorisierbarer Operator von einem $(B, V)$-Raum $X$ in einen $(B, V, b v)$-Raum $Y$, so ist $T^{+}=T^{r+}, T^{-}=T^{r-}$ und $|T|=T^{(\sim)}$.

BEweIs. Nach Konstruktion ist $T^{r+} \geqq 0$ und $T^{r+} \geqq T$. Ist $S \geqq 0$ und $S \geqq T$, so ist

$$
Q^{r+}(Z(f), T)=\sum_{i=1}^{k}\left(f_{i} T\right)^{+} \leqq \sum_{i=1}^{k}\left(f_{i} S\right)^{+}=f S
$$

und also $T^{r+} \leqq S$, das heißt $T^{+}=T^{r+}$. Ebenso zeigt man $T^{r-}=T^{-}$. Aus

$$
\left|f_{i} T\right|=\left(f_{i} T\right)^{+}+\left(-f_{i} T\right)^{+}
$$

folgt $T^{(\sim)}=T^{r+}+T^{r-}=|T|$

Definition 3.1. Für majorisierbare Operatoren von einem $(K o, B)$ Raum $X$ in einen $(K o, B, b v)$-Raum $Y$ setzen wir: $|T|=T^{(\sim)}$ und nennen $|T|$ den Absolutbetrag des Operators $T$.

Bemerkungen. (1) Im Fall $X=L_{1}(\Omega, B, m)$ ist $|T|$ auch bei Beschränkung auf solche Zerlegungen $\left\{f_{1}, \ldots, f_{k}\right\}=\left\{f \chi_{A_{1}}, \ldots, f \chi_{A_{k}}\right\}$, die aus einer meßbaren Zerlegung $\left\{A_{1}, \ldots, A_{k}\right\}$ von $\Omega$ hervorgehen, konstruierbar (R. V. Chacon and U. Krengel [5]). Satz 3.2 wird zeigen, daß der dort konstruierte Operator $|T|$ identisch mit dem dieser Arbeit ist.

(2) Gegenüber der in Satz 2.1 beschriebenen Konstruktion von $|T|$ hat die von $T^{(\sim)}$ außer dem Vorteil der Übertragbarkeit ins Komplexe noch den folgenden: Ist $Y$ ein $(K o, B, m K)$-Raum, so gilt, wenn $Z(f)$ das aufsteigend gefilterte System $B(f)$ durchläuft,

$$
\left\|f T^{(\sim)}-Q^{(\sim)}(Z(f), T)\right\| \rightarrow 0 .
$$

Wir können in diesem Fall also schreiben

$$
f|T|=\lim _{Z(f) \in B(f)} Q^{(\sim)}((Z f), T) \quad(\text { Norm }) .
$$

Nun bereiten wir eine weitere Darstellung von $|T|$ vor:

Lemma 3.1. Sei $T_{0}$ eine Majorante des den $(K o, B)-R a u m ~ X$ in den $(K o, B)$-Raum $Y$ abbildenden Operators $T$. Dann ist für $|g| \leqq f \in X^{+}$stets $|g T| \leqq f T_{0}$, und ist $Y$ ein $(K o, B, b v)$-Raum, so ist $|T| \leqq T_{0}$ auch eine Majorante von $T$. 
Beweis. Sei $g \in X$ und für $h_{k} \in X$ gelte $\left\|h_{k}-g\right\| \rightarrow 0$ für $k \rightarrow \infty$. Sei ferner $h_{k}=\sum_{i=1}^{n(k)} a_{i k} h_{i k}, \quad\left|a_{i k}\right|=1, \quad h_{i k} \in X^{+}$und $h_{i k} \wedge h_{j k}=0, \quad i \neq j$, also $h_{k} \in \operatorname{Tr}(X)$. Bilden wir

so ist

$$
g_{i k}=h_{i k} \wedge|g| \quad \text { und } \quad g_{k}=\sum_{i=1}^{n(k)} a_{i k} g_{i k}
$$

$$
\left\|h_{k}-g_{k}\right\|=\left\|\left|h_{k}\right|-\left(\left|h_{k}\right| \wedge|g|\right)\right\| .
$$

Aus $h_{k} \rightarrow g$ folgt $\left|h_{k}\right| \rightarrow|g|$ und $\left|h_{k}\right| \wedge|g| \rightarrow|g|$, also $\left\|h_{k}-g_{k}\right\| \rightarrow 0$. Es gibt demnach zu jedem $g \in X$ Elemente $g_{k} \in \operatorname{Tr}(X)$ mit $g_{k} \rightarrow g$ und $\left|g_{k}\right| \leqq|g|$. Nun ist

$$
\begin{aligned}
\left|g_{k} T\right|=\left|\sum_{i=1}^{n(k)} a_{i k} g_{i k} T\right| & \leqq \sum_{i=1}^{n(k)}\left|a_{i k}\right|\left|g_{i k} T\right| \\
& \leqq \sum_{i=1}^{n(k)} g_{i k} T_{0} \leqq|g| T_{0} \leqq f T_{0} .
\end{aligned}
$$

Durch Approximation folgt $|g T| \leqq f T_{0}$. Die letzte Behauptung ergibt sich aus der Konstruktion von $T^{(\sim)}$.

SATz 3.2. Ist $T$ ein majorisierbarer Operator von einem $(K o, B)-R a u m X$ in einen $(K o, B, b v)$-Raum $Y$, so ist für $f \in X^{+}$

$$
f|T|=\sup _{|g| \leqq f}|g T|=\sup _{|g| \leqq f} \operatorname{Re}(g T) .
$$

BeweIs. Nach Lemma 3.1 und Formel (1.6) gilt $f(T) \geqq|g T| \geqq \operatorname{Re}(g T)$ für jedes $|g| \leqq f$. Die Suprema existieren daher, und bezeichnet man die rechte Seite in (3.6) mit $f T^{a}$, so gilt $|T| \geqq T^{a}$. Ist $T^{a} \geqq|T|$ falsch, so gibt es ein $f \in X^{+}$und eine Zerlegung $Z(f)=\left\{f_{1}, \ldots, f_{k}\right\}$, für die

$$
\left(\sum_{i=1}^{k}\left|f_{i} T\right|-f T^{a}\right)^{+}=p>0
$$

ist. Wir setzen $p_{0}=\frac{1}{2} p / k>0$. Es gilt

$$
\left|f_{i} T\right|=\sup _{\left|a_{i}\right|=1} \operatorname{Re}\left(a_{i} f_{i} T\right)
$$

Daher gibt es für $i=1$ ein $a_{i}$ mit $\left|a_{i}\right|=1$ und

$$
\left(\operatorname{Re}\left(a_{i} f_{i} T\right)-\left(\left|f_{i} T\right|-p_{i-1}\right)\right)^{+}=p_{i}>0 .
$$

Aus (3.8) folgt zugleich $p_{i} \leqq p_{i-1}$. Sukzessive bestimmen wir so $a_{1}, \ldots, a_{k}$ und $p_{0} \geqq p_{1} \geqq \ldots \geqq p_{k}>0$. Wir zeigen für $g_{1}, g_{2} \in \operatorname{Re}(X)$ die Beziehung

$$
g_{1}{ }^{+} \geqq g_{2}{ }^{+} \quad \text { impliziert } \quad\left(g_{1}+g_{2}\right)^{+} \geqq g_{2}{ }^{+} \text {. }
$$


Aus $g_{1}{ }^{+} \geqq g_{2}{ }^{+}$und $g_{1}{ }^{+} \wedge g_{1}{ }^{-}=0$ folgt $g_{2}{ }^{+} \wedge g_{1}{ }^{-}=0$, und zusammen mit $g_{2}{ }^{+} \wedge g_{2}{ }^{-}=0$ folgt $g_{2}{ }^{+} \wedge\left(g_{1}{ }^{-}+g_{2}^{-}\right)=0$. Nun ist aber

$$
\begin{aligned}
\left(g_{1}+g_{2}\right)^{+} & =\left(g_{1}^{+}+g_{2}^{+}-\left(g_{1}^{-}+g_{2}^{-}\right)\right)^{+} \\
& \geqq\left(g_{2}^{+}-\left(g_{1}^{-}+g_{2}^{-}\right)\right)^{+}=g_{2}^{+} .
\end{aligned}
$$

Für $r=1$ ist (3.9) gleichbedeutend mit

$$
\left(\sum_{i=1}^{r} \operatorname{Re}\left(a_{i} f_{i} T\right)-\sum_{i=1}^{r}\left(\left|f_{i} T\right|-p_{i-1}\right)\right)^{+} \geqq p_{r}>0 .
$$

Durch Anwendung von (3.10) können wir für $r=1, \ldots, k-1$ von $r$ auf $r+1$ schließen. Setzt man

$$
h=\sum_{i=1}^{k} \operatorname{Re}\left(a_{i} f_{i} T\right)-\sum_{i=1}^{k}\left(\left|f_{i} T\right|-p_{i-1}\right),
$$

so folgt also $h^{+} \geqq p_{k}>0$. Setzt $\operatorname{man} g=\sum_{i=1}^{k} a_{i} f_{i}$, so ist $|g| \leqq \sum_{i=1}^{k} f_{i}=f$. Daher ist $\sum_{i=1}^{k} \operatorname{Re}\left(a_{i} f_{i} T\right) \leqq f T^{a}$. Wir erhalten aus (3.7) und $\sum_{i=1}^{k} p_{i-1} \leqq \frac{1}{2} p$ die Abschätzung

$$
\begin{aligned}
h^{-}=(-h)^{+} & =\left(\sum_{i=1}^{k}\left|f_{i} T\right|-\sum_{i=1}^{k} p_{i-1}-\sum_{i=1}^{k} \operatorname{Re}\left(a_{i} f_{i} T\right)\right)^{+} \\
& \geqq\left(\sum_{i=1}^{k}\left|f_{i} T\right|-\frac{1}{2} p-f T^{a}\right)^{+} \geqq \frac{1}{2} p .
\end{aligned}
$$

Dies führt zu $h^{+} \wedge h^{-} \geqq p_{k} \wedge \frac{1}{2} p=p_{k}>0$, also einem Widerspruch. Damit ist auch $T^{a} \geqq|T|$ bewiesen.

Bemerkungen. (1) Ein (3.6) entsprechendes Resultat in bedingt vollständigen Vektorverbänden ohne jede Metrik folgt sehr leicht aus (3.1). (Kantorovitch [14, S. 222].)

(2) Mit dem Ansatz (3.6) haben Dunford-Schwartz [8], [7, S. 672] gezeigt: Ist $(\Omega, B, m)$ ein $\sigma$-endlicher Maßraum, $X=L_{1}(\Omega, B, m)=Y, T$ ein Operator von $X$ nach $Y$ mit $\|T\|_{1} \leqq K_{1}<\infty$ und $\|T\|_{\infty} \leqq K_{\infty}<\infty$, so existiert ein positiver Operator $|T|$ mit den gleichen Eigenschaften, für den $|f||T| \geqq|f T|, f \in L_{1}$, ist. Dieses folgt einfacher aus (3.6) und Satz 4.1 dieser Arbeit.

(3) Der Beweis zeigt zugleich: Will man beweisen, daß $T$ majorisierbar ist, so kann dazu (3.6) dienen: Ist $f T^{a} \in Y^{+}$für jedes $f \in X^{+}$definiert, so ist für jedes $Z(f)$

$$
Q^{(\sim)}(Z(f), T) \leqq f T^{a} .
$$

$f T^{a}$ kann dann die Rolle von $f T_{0}$ in der Konstruktion von $f T^{(\sim)}$ übernehmen, wenn außerdem für eine Konstante $C<\infty$ und alle $f \in X^{+}$die Ungleichung $\left\|f T^{a}\right\| \leqq C\|f\|$ gilt. 
Wir wollen noch feststellen, daß $|T|$ auch die gleiche Darstellung (1.6) besitzt wie der Absolutbetrag von Elementen aus $(K o, B)$-Räumen. Dazu definieren wir den Operator $T a$ durch

$$
f(T a)=(f T) a .
$$

Für einen Operator $S$ bedeute ferner $\operatorname{Re}(S)$ den Operator, der $f \in X^{+}$in $\operatorname{Re}(f S)$ abbildet. Nun gilt:

SATZ 3.3. Ist $T$ ein majorisierbarer Operator von einem $(K o, B)-R a u m$ $X$ in einen $(K o, B, b v)$-Raum $Y$, so gilt

$$
|T|=\sup _{|a|=1} \operatorname{Re}(T a) .
$$

BeweIs. Das Supremum reeller Operatoren wird nach Formel (2.2) wie in $(B, V, b v)$-Räumen gebildet. Die rechte Seite von (3.12) bezeichnen wir mit ]T[. Für $f \in X^{+}$, gegebenes $Z(f)$ und $\left|a_{1}\right|=\ldots=\left|a_{k}\right|=1$ ist

$$
\begin{aligned}
\sum_{i=1}^{k} f_{i} \operatorname{Re}\left(T a_{i}\right) & =\sum_{i=1}^{k} \operatorname{Re}\left(f_{i} T a_{i}\right) \\
& \leqq \sum_{i=1}^{k}\left|f_{i} T\right| \leqq \sum_{i=1}^{k} f_{i}|T|=f|T|
\end{aligned}
$$

Daraus folgt $] T[\leqq|T|$. Andererseits folgt durch Anwendung der Ungleichung

$$
\begin{aligned}
f] T[ & =\sup _{\left|a_{i}\right|=1, Z(f)} \sum_{i=1}^{k} f_{i} \operatorname{Re}\left(T a_{i}\right) \\
& \geqq \sup _{|a|=1, Z(f)} \sum_{i=1}^{k} f_{i} \operatorname{Re}(T a) \\
& =\sup _{|a|=1} f \operatorname{Re}(T a)=\sup _{|a|=1} \operatorname{Re}(a(f T))=|f T|
\end{aligned}
$$

auf die $f_{i}$ eines $Z(f)$, daß

$$
\left.\sum_{i=1}^{k}\left|f_{i} T\right| \leqq f\right] T[
$$

Aus (3.4) ergibt sich damit $] T[\geqq|T|$.

\section{Majorisierbarkeitssätze.}

In diesem Abschnitt lernen wir Paare von Räumen $X$ und $Y$ kennen, für die alle stetigen Operatoren von $X$ nach $Y$ majorisierbar sind.

Lemma 4.1. Sei $X$ ein (Ko,B)-Raum und $Y$ ein $(K o, B, b v)$-Raum. Sind $T, T_{1}$ und $T_{2}$ majorisierbare Operatoren von $X$ nach $Y$ und ist a skalar, so gilt: 
a) Es ist $|T| \geqq 0 ; T \geqq 0$ impliziert $T=|T|$.

b) $|T a|=|T||a|$.

c) $\left|T_{1}+T_{2}\right| \leqq\left|T_{1}\right|+\left|T_{2}\right|$.

d) ||$T_{1}|-| T_{2}|| \leqq\left|T_{1}-T_{2}\right|$.

e) Ist zudem $X=Y$, so gilt $|f||T|^{n} \geqq\left|f T^{n}\right|$.

BeweIs. (a), (b) und (c) folgen unmittelbar aus der Definition (3.4) von $T^{(\sim)}$. (e) folgt durch Induktion aus Lemma (3.1). Aus (c) folgt

$$
\left|T_{1}\right|-\left|T_{2}\right| \leqq\left|T_{1}-T_{2}\right|+\left|T_{2}\right|-\left|T_{2}\right|=\left|T_{1}-T_{2}\right|
$$

und ebenso

$$
\left|T_{2}\right|-\left|T_{1}\right| \leqq\left|T_{1}-T_{2}\right|
$$

Da $\left|T_{1}\right|,\left|T_{2}\right|$ und $\left|T_{1}-T_{2}\right|$ reelle majorisierbare Operatoren sind und diese einen Verband bilden, ist

$$
|| T_{1}|-| T_{2}||=\left(\left|T_{1}\right|-\left|T_{2}\right|\right) \vee\left(\left|T_{2}\right|-\left|T_{1}\right|\right) \leqq\left|T_{1}-T_{2}\right|
$$

SATz 4.1. Ist $X$ ein $(K o, B, L)$-Raum und $Y$ ein $(K o, B, m K)$-Raum, so sind alle stetigen Operatoren von $X$ nach $Y$ majorisierbar und es ist $\||T|\|=\|T\|$. Die Abbildung $T \rightarrow|T|$ ist normstetig.

Beweis. Sei $f \in X^{+}$und $Z(f)=\left\{f_{1}, \ldots, f_{k}\right\}$. Wir definieren $Q^{(\sim)}(Z(f), T)$ durch (3.3) ohne Abschätzung durch $f T_{0}$. Die Eigenschaft $(L)$ gestattet die Abschätzung

$$
\left\|Q^{(\sim)}(Z(f), T)\right\|=\left\|\sum_{i=1}^{k}\left|f_{i} T\right|\right\| \leqq \sum_{i=1}^{k}\left\|f_{i}\right\|\|T\|=\|f\|\|T\| \cdot
$$

Wir wissen, daß $Q^{(\sim)}(Z(f), T)$ monoton wächst, wenn $Z(f)$ das aufsteigend gefilterte System $3(f)$ durchläuft. Wenden wir nun in $Y$ den Satz von der monotonen Konvergenz an, so sehen wir: Es existiert

$$
f T^{(\sim)}=\lim _{Z(f) \in B(f)} Q^{(\sim)}(Z(f), T)
$$

als Normlimes. Zugleich folgt

$$
\left\|f T^{(\sim)}\right\| \leqq\|f\|\|T\|
$$

$T^{(\sim)}$ läßt sich also zu dem stetigen Operator $|T|$ mit $\||T|\| \leqq\|T\|$ fortsetzen. $\||T|\| \geqq\|T\|$ wiederum folgt aus Satz 3.2. Ist $\left\|T-T_{k}\right\|<\varepsilon$, so ist daher nach Lemma 4.1 (d)

$$
\begin{aligned}
\left\||T|-\left|T_{k}\right|\right\| & =\left\||| T|-| T_{k}||\right\| \\
& \leqq\left\|T-T_{k} \mid\right\|=\left\|T-T_{k}\right\|<\varepsilon
\end{aligned}
$$

und die Abbildung $T \rightarrow|T|$ normstetig. 
Corollar 4.1. Die stetigen linearen Operatoren $T$ von einem $(L)$-Raum $X$ in einen $(B, V, m K)$-Raum bilden einen $(B, V, b v)$-Raum.

Bewers. Die Majorisierbarkeit aller Operatoren folgt wie im komplexen Fall, ebenso die Normstetigkeit des Übergangs zum Absolutbetrag. Da $T^{+}=\frac{1}{2}(|T|+T)$ ist, ist auch der Übergang zu $T^{+}$normstetig und es ist

$$
\left\|T^{+}\right\| \leqq \frac{1}{2}(\||T|\|+\|T\|)=\|T\| \text {. }
$$

$\||T|\|=\|T\|$ bedeutet insbesondere, daß die Norm symmetrisch ist, d. h., daß für $T_{1} \wedge T_{2}=0$ stets $\left\|T_{1}-T_{2}\right\|=\left\|T_{1}-T_{2} \mid\right\|=\left\|T_{1}+T_{2}\right\|$ ist. $\quad(b v)$ folgt aus Satz 2.1.

Bemerkung. Ein zu diesem Corollar im wesentlichen äquivalenter Satz ist schon 1940 von Kantorovitch [14, Satz 11] bewiesen worden. Davon unabhängig wurde 1962 von R. V. Chacon und dem Autor [5] ein Spezialfall von Satz 4.1 gefunden.

SATz 4.2. Ist $X$ ein $(K o, B)$-Raum und $Y$ ein $(K o, B, b v, 1)-R a u m$, so sind alle stetigen linearen Operatoren $T$ von $X$ nach $Y$ majorisierbar. Es ist $\||T|\|=\|T\|$ und also die Abbildung $T \rightarrow|T|$ normstetig.

BeweIs. Ist $T$ ein stetiger Operator und $f \in X^{+}$, so setzen wir

$$
f T^{b}=\sup _{|g| \leqq f}|g T|, \quad f T^{a}=\sup _{|g| \leqq f} \operatorname{Re}(g T) .
$$

Aus

folgt

$$
\|g T\| \leqq\|g\|\|T\| \leqq\|f\|\|T\|
$$

$$
|g T| \leqq\|f\|\|T\| 1, \quad|g| \leqq f .
$$

Daher ist $f T^{b} \in Y^{+}$definiert, und aus $|g T|=\sup _{|a|=1} \operatorname{Re}(a g T)$ und $|a g| \leqq f$, wenn $|a|=1$, ergibt sich $f T^{a}=f T^{b}$. Zugleich folgt aus $f T^{a} \leqq\|f\|\|T\| 1$ die Abschätzung $\left\|f T^{a}\right\| \leqq\|f\|\|T\|$. Nach Bemerkung 3) zu Satz (3.2) genügt das, um $f T^{(\sim)}$ zu konstruieren und zwar mit

$$
\left\|f T^{(\sim)}\right\| \leqq\left\|f T^{a}\right\| \leqq\|f\|\|T\| \text {. }
$$

Wir erhalten zu $T$ den Absolutbetrag $|T|=T^{(\sim)}$ mit $\||T|\|=\|T\|$. Wie im Beweis von Satz 4.1 folgt aus Lemma 4.1 (d) die Normstetigkeit der Abbildung $T \rightarrow|T|$.

Bemerkung. Da der Banachraum $Y$ der komplexen Zahlen ein $(K o, B, b v, 1)$-Raum ist, sind insbesondere stetige lineare Funktionale majorisierbar. Als Spezialfall der Aussage von Satz 4.2 für reelle Banachverbände ergibt sich, daß die stetigen Operatoren in dem $(M)$-Raum $M(\Omega)$ (reell) und dem $(M)$-Raum $L_{\infty}(\Omega, B, m)$ (reell) für $\sigma$-endliches $m$ 
einen Verband bilden. Wegen der Dualität von $(L)$-Räumen und $(M)$ Räumen (Kakutani [12, S. 1021]) liegt die Vermutung nahe, daß die stetigen Operatoren in $(M)$-Räumen einen Verband bilden. Wir betrachten ein Gegenbeispiel, das zugleich zeigt, daß majorisierbare Operatoren $T$ nicht einen positiven Teil $T^{+}$zu besitzen brauchen, wenn der Bildraum $Y$ nicht ein bedingt vollständiger Verband ist.

Beispiel 5. In dem abgeschlossenen Intervall $[-1,+1]=X=Y$ definieren wir eine monoton wachsende Folge von Funktionen

$$
g_{k}(x)=0 \vee(k x) \wedge 1, \quad k=0,1, \ldots
$$

Es ist $g_{k} \in C(X)$ (vgl. Beispiel (4)). Es sei

$$
h_{0}=g_{0} \equiv 0, \quad h_{k}=g_{k}-g_{k-1}, \quad k=1,2, \ldots .
$$

Dann ist $h_{i} \geqq 0$ und $\sum_{i=0}^{n} h_{i}=g_{n}$. In $[-1,0]$ ist $g_{n}(x)=0$ und strebt nach 1 in $(0,+1]$. Sei ferner $a_{0}<a_{1}<a_{2}<\ldots$ eine streng monotone Folge in $[-1,+1]$ und $\lim _{n \rightarrow \infty} a_{n}=a$. Für $f \in C(X)$ bilden wir

und

$$
f T_{n}(x)=\sum_{i=0}^{n} f\left(a_{2 i}\right) h_{i}(x)-\sum_{i=0}^{n} f\left(a_{2 i+1}\right) h_{i}(x)
$$

$$
f T(x)=\lim _{n \rightarrow \infty} f T_{n}(x),
$$

wobei der Limes punktweise zu verstehen ist: Für $x \in[-1,0]$ ist $f T_{n}(x)=0$, für $\geqq 1 / i$ ist $g_{j}(x)=1$ für alle $j \geqq i$ und also $h_{j}(x)=0$ für $j \geqq i+1$. Der Limes existiert also überall. Ist $\varepsilon>0$ vorgegeben, so gibt es ein solches $N(\varepsilon)$, daß

Dann ist

$$
\left|f\left(a_{n}\right)-f(a)\right|<\frac{1}{4} \varepsilon \quad \text { für } \quad n \geqq N(\varepsilon) .
$$

$\left|\sum_{i=n+1}^{m} f\left(a_{2 i}\right) h_{i}(x)-\sum_{i=n+1}^{m} f\left(a_{2 i+1}\right) h_{i}(x)\right| \leqq \sum_{i=n+1}^{m}\left|f\left(a_{2 i}\right) h_{i}(x)-f\left(a_{2 i+1}\right) h_{i}(x)\right|<\frac{1}{2} \varepsilon$

für $m>n$ und jedes $x$. Da $f T_{n}(x)$ stetig ist, ist also $f T(x)$ überall stetig, das heißt $T$ ist eine Abbildung von $C(X)$ in $C(X)$. T ist linear und es gilt $\|T\| \leqq 2$. Ist nämlich $\|f\|=\sup _{x \in X}|f(x)|=M$, so ist

$$
\left|f T_{n}(x)\right| \leqq 2 M g_{n}(x) \leqq 2 M .
$$

Bilden nun die stetigen linearen Operatoren auf $C(X)$ einen Verband, so läßt sich $T^{+}=T \vee 0$ bilden. Wie kann $T^{+}$aussehen? Auf $X$ konstruieren wir stetige Funktionen $p_{n}(x), n \geqq 0$, für die $0 \leqq p_{n}(x) \leqq 1, p_{n}\left(a_{2 i}\right)=1$, $i=0, \ldots, n$, und $p_{n}\left(a_{2 i+1}\right)=0, i=0, \ldots, n$, und $p_{n}(x)=0$ für $x \geqq a_{2 n+1}$ ist. 
Dann ist für $f \geqq 0$

für jedes $n$, also

$$
\begin{aligned}
f T^{+}=f p_{n} T^{+}+f\left(1-p_{n}\right) T^{+} & \geqq f p_{n} T^{+} \\
& \geqq f p_{n} T=\sum_{i=0}^{n} f\left(a_{2 i}\right) h_{i}(x)
\end{aligned}
$$

$$
f T^{+} \geqq \sum_{i=0}^{\infty} f\left(a_{2 i}\right) h_{i} .
$$

Andererseits ist $f T^{+}(x)=0$ auf $[-1,0)$. Wäre etwa $f T^{+}(\lambda) \neq 0$ für $\lambda \in$ $[-1,0)$ und $f \geqq 0$, also $f T^{+}(\lambda)>0$, so konstruieren wir ein $q(x) \in C(X)$ mit $0 \leqq q(x) \leqq 1, q(\lambda)=0$ und $q(x)=1$ auf $[0,1]$. Dann ist durch

$$
(f S)(x)=\left(f T^{+}\right)(x) q(x)
$$

ein stetiger linearer Operator $S$ von $C(X)$ in $C(X)$ gegeben, der $S \geqq 0$ und $S \geqq T$ genügt, für den aber nicht $T^{+} \leqq S$ gilt.

Ist nun speziell $f \equiv 1$, so erhalten wir $f T^{+}(x)=0$ auf $[-1,0)$ und aus (4.3) $f T^{+}(x) \geqq 1$ auf $(0,1]$, einen Widerspruch dazu, daß $f T^{+}$zu $C(X)$ gehören muß.

Dabei ist $T$ majorisierbar durch den folgenden Operator $T^{(m)}$ :

$f T^{(m)}(x)=\left\{\begin{array}{l}\sum_{i=0}^{\infty}\left\{f\left(a_{2 i}\right)\left(h_{i}(x)+h_{i}(-x)\right)+f\left(a_{2 i+1}\right)\left(h_{i}(x)+h_{i}(-x)\right)\right\} \text { für } x \neq 0, \\ 2 f(a) \quad \text { für } x=0 .\end{array}\right.$

Der Beweis der Stetigkeit und Linearität von $T^{(m)}$ und von $f T^{(m)} \in C(X)$ läuft wie der entsprechende Beweis für $T$.

Bemerkung. Da die Schwierigkeit nur daher rührt, daß $f T^{+}$unstetig ist, können wir sie dadurch umgehen, daß $T$ als Operator von $C(X)$ in $M(X) \supseteqq C(X)$ betrachtet wird. Ähnlich gehen wir in beliebigen $(M)$ Räumen vor:

Defrnition 4.1. Zwei $(K o, B)$-Räume bzw. $(B, V)$-Räume $X$ und $X^{\prime}$ heißen isomorph, wenn eine eineindeutige strukturtreue Abbildung $\varphi$ von $X$ auf $X^{\prime}$, ein sog. Isomorphismus $\varphi$ existiert. Strukturtreue besagt:

$$
(f+g) \varphi=f \varphi+g \varphi, \quad(\alpha f) \varphi=\alpha(f \varphi), \quad\|f\|=\|f \varphi\|, \quad|f \varphi|=|f| \varphi,
$$

$f \leqq g$ impliziert $f \varphi \leqq g \varphi$ und umgekehrt, für $(K o, B)$-Räume ferner $\operatorname{Re}(f) \varphi=\operatorname{Re}(f \varphi)$.

Sind $\varphi_{x}$ und $\varphi_{y}$ Isomorphismen von $X$ mit $X^{\prime}$, bzw. von $Y$ mit $Y^{\prime}$ und ist $T$ ein stetiger linearer Operator von $X$ nach $Y$, so ist $T^{\prime}=\varphi_{x}{ }^{-1} T \varphi_{\nu}$ ein stetiger linearer Operator von $X^{\prime}$ nach $Y^{\prime}$. Ist $T_{0}$ eine Majorante von 
$T$, so ist $T_{0}{ }^{\prime}=\varphi_{x}{ }^{-1} T_{0} \varphi_{y}$ eine Majorante von $T^{\prime}$. Es gilt mit den eingeführten Bezeichnungen:

Lemma 4.2. Ist $Y$ ein $(K o, B, b v)$-Raum, so ist $Y^{\prime}=Y \varphi_{y}$ ein $(K o, B, b v)$ Raum und

$$
\left|\varphi_{x}{ }^{-1} T \varphi_{y}\right|=\varphi_{x}{ }^{-1}|T| \varphi_{y}
$$

für jeden majorisierbaren Operator $T$.

Beweis. Da $\varphi_{y}$ eine eineindeutige Abbildung von $Y$ auf $Y^{\prime}$ ist, sind auch beliebige Supremumsbildungen mit dem Operator $\varphi_{y}$ vertauschbar. Die Zerlegungen $Z(f)$ eines $f \in X^{+}$entsprechen eineindeutig Zerlegungen $Z\left(f \varphi_{x}\right)=(Z(f)) \varphi_{x}$. Es ist

$$
Q^{(\sim)}\left(Z\left(f \varphi_{x}\right), \varphi_{x}{ }^{-1} T \varphi_{y}\right)=\sum_{i=1}^{k}\left|f_{i} \varphi_{x} \varphi_{x}{ }^{-1} T \varphi_{y}\right|=\sum_{i=1}^{k}\left|f_{i} T\right| \varphi_{y} .
$$

Hieraus folgt $f \varphi_{x}\left|\varphi_{x}{ }^{-1} T \varphi_{y}\right|=f|T| \varphi_{y}$ und also die Behauptung.

Kakutani [13, S. 998] hat gezeigt, daß zu jedem ( $M)$-Raum $Y$ ein kompakter Hausdorffraum $\Omega$ existiert, für den $Y$ in $C(\Omega)$, insbesondere also in $M(\Omega)$ isomorph eingebettet werden kann. Vollzieht man die in der Algebra übliche Identifizierung von $h \in Y$ mit $h \varphi_{y} \in M(\Omega)$, so können wir sagen:

Satz 4.3. Ist $X$ ein $(B, V)$-Raum und $Y$ ein (M)-Raum, so kann $Y$ derart in einen $(B, V, b v, 1)$-Raum $Y^{\prime} \supseteqq Y$ eingebettet werden, da $\beta$ jeder stetige lineare Operator $T$ von $X$ nach $Y$ einen Absolutbetrag $|T|$ besitzt, der ein stetiger linearer Operator von $X$ nach $Y^{\prime}$ ist.

Der folgende Majorisierbarkeitssatz für zusammengesetzte Operatoren ist vor allem im Fall $X=Y=Z$ wichtig:

Satz 4.4. Sind $X, Y$ und $Z(K o, B)$-Räume und ist $T_{1}$ ein majorisierbarer Operator von $X$ nach $Y$ und $T_{2}$ ein majorisierbarer Operator von $Y$ nach $Z$, so ist die Hintereinanderausführung $T_{1} T_{2}$ majorisierbar. Sind $Y$ und $Z$ sogar $(K o, B, b v)$-Räume, so ist

$$
\left|T_{1} T_{2}\right| \leqq\left|T_{1}\right|\left|T_{2}\right|
$$

BeweIs. $T_{01}$ und $T_{02}$ seien Majoranten von $T_{1}$ und $T_{2}$. Für $f \in X^{+}$ist dann $f T_{01} \geqq\left|f T_{1}\right|$ und nach Lemma 3.1 ist $f T_{01} T_{02} \geqq\left|f T_{1} T_{2}\right|$, das heißt $T_{01} T_{02}$ ist eine Majorante von $T_{1} T_{2}$. Ist $Z(f)=\left\{f_{1}, \ldots, f_{k}\right\}$, so ist 
und also $\left|T_{1} T_{2}\right| \leqq\left|T_{1}\right|\left|T_{2}\right|$.

$$
\begin{aligned}
Q^{(\sim)}\left(Z(f), T_{1} T_{2}\right) & =\sum_{i=1}^{k}\left|f_{i} T_{1} T_{2}\right| \\
& \leqq \sum_{i=1}^{k}\left|f_{i} T_{1}\right|\left|T_{2}\right| \\
& \leqq \sum_{i=1}^{k} f_{i}\left|T_{1}\right|\left|T_{2}\right|=f\left|T_{1}\right|\left|T_{2}\right|
\end{aligned}
$$

\section{Operatoren in $L_{2}(\Omega, B, m)$.}

Die linearen stetigen Operatoren in $L_{2}(\Omega, B, m)$ bilden zwar nach Satz 2.1 einen bedingt vollständigen Verband, sie bilden aber im allgemeinen keinen Verband. Wir wollen dies an einem Beispiel einsehen und dann daraus ein einfaches Kriterium für die Verbandseigenschaft in $L_{2}$ herleiten.

Beispiel 6. Sind $\left(\Omega_{n}, B_{n}, m_{n}\right), n=1,2, \ldots$, Maßräume, so kann man sie als Bestandteile einer disjunkten Zerlegung eines Maßraumes $\left(\Omega^{\prime}, B^{\prime}, m^{\prime}\right)$ auffassen. Für $f \in L_{2}\left(\Omega^{\prime}, B^{\prime}, m^{\prime}\right)$ sei $f \varphi_{n}$ die Einschränkung von $f$ auf $\Omega_{n}$, und für $f_{n} \in L_{2}\left(\Omega_{n}, B_{n}, m_{n}\right)$ sei

$$
f_{n} \psi_{n}(\omega)=\left\{\begin{array}{ll}
f_{n}(\omega) & \text { für } \omega \in \Omega_{n}, \\
0 & \text { für } \omega \notin \Omega_{n}
\end{array} .\right.
$$

Ist in jedem $L_{2}\left(m_{n}\right)=L_{2}\left(\Omega_{n}, B_{n}, m_{n}\right)$ ein stetiger linearer Operator $T_{n}$ gegeben, so ist durch

$$
f T^{\prime}(\omega)=\sum_{i=1}^{\infty} f \varphi_{n} T_{n} \psi_{n}
$$

ein linearer Operator $T^{\prime}$ von $L_{2}\left(\Omega^{\prime}, B^{\prime}, m^{\prime}\right)$ in den Raum der $B^{\prime}$-meßbaren Funktionen gegeben. Ist $\sup \left\|T_{n}\right\|_{2}=K_{0}<\infty$, so ist $T^{\prime}$ ein stetiger Operator in $L_{2}\left(m^{\prime}\right)$ mit $\left\|T^{\prime}\right\|_{2}=K_{0}$ : Ist nämlich $f \in L_{2}\left(m^{\prime}\right)$, so ist

$$
\|f\|_{2}^{2}=\sum_{n=1}^{\infty}\left\|f \varphi_{n}\right\|_{2}^{2} \quad \text { und } \quad\left\|f T^{\prime}\right\|_{2}^{2}=\sum_{n=1}^{\infty}\left\|f \varphi_{n} T_{n}\right\|_{2}^{2} .
$$

Aus $\left\|T_{n}\right\|_{2} \leqq K_{0}$ folgt $\left\|f \varphi_{n} T_{n}\right\|_{2}{ }^{2} \leqq K_{0}{ }^{2}\left\|f \varphi_{n}\right\|_{2}{ }^{2}$ und also $\left\|f T^{\prime}\right\|_{2}{ }^{2} \leqq K_{0}{ }^{2}\|f\|_{2}{ }^{2}$, und daraus ergibt sich $\left\|T^{\prime}\right\|_{2} \leqq K_{0}$. Daß umgekehrt für jedes $n$ die Ungleichung $\left\|T^{\prime}\right\|_{2} \geqq\left\|T_{n}\right\|_{2}$ gilt, sieht man durch Betrachtung der Funktionen $f \in L_{2}\left(m^{\prime}\right)$, die außerhalb von $\Omega_{n}$ den Wert 0 haben. Strebt $\left\|T_{n}\right\|_{2}$ nach $\infty$, so ist demnach $T$ kein stetiger Operator. Existieren stetige Operatoren $T_{n}{ }^{+}$und $T^{\prime+}$, so ist

$$
f T^{\prime}+(\omega)=\sum_{i=1}^{\infty} f \varphi_{n} T_{n}{ }^{+} \psi_{n}
$$

Es genügt also, Maßräume $\left(\Omega_{n}, B_{n}, m_{n}\right)$ und lineare Operatoren $T_{n}$ mit 
$\left\|T_{n}\right\|_{2}=1$ so anzugeben, daß $T_{n}^{+}, T_{n}^{-}$und $\left|T_{n}\right|$ als stetige Operatoren in $L_{2}\left(m_{n}\right)$ existieren, jedoch $\left\|\mid T_{n}\right\|_{2} \rightarrow \infty$ gilt.

$\Omega_{n}$ bestehe aus $2^{n}$ Punkten $x_{n i}: i=1,2, \ldots 2^{n}, B_{n}$ aus allen Teilmengen von $\Omega_{n}$ und $m_{n}$ erteile jedem Punkt das Maß 1. Induktiv erklären wir eine Hilfsmatrix $A_{n}$ :

$$
A_{1}=\left(\begin{array}{rr}
+1 & +1 \\
+1 & -1
\end{array}\right), \quad A_{j+1}=\left(\begin{array}{cc}
A_{j} & A_{j} \\
A_{j} & -A_{j}
\end{array}\right) .
$$

In $A_{j}=\left(a_{i k}\right)$ sind die $a_{i k}$ unabhängig von $j$, da beim Schritt von $j$ nach $j+1$ die schon definierte Matrix nur vergrößert wird. Die $a_{i k}$ nehmen nur die Werte \pm 1 an. Ferner ist für $1 \leqq k^{\prime}, k^{\prime \prime} \leqq 2^{j}$

$$
\sum_{i=1}^{2 j} a_{i k^{\prime}} a_{i k^{\prime \prime}}=2^{j} \delta_{k^{\prime} k^{\prime \prime}} \text {, }
$$

wobei $\delta_{k^{\prime} k^{\prime \prime}}$ das Kronecker-Symbol ist. Für $j=1$ oder $k^{\prime}=k^{\prime \prime}$ ist das trivial. Sei nun ohne Beschränkung der Allgemeinheit $k^{\prime}<k^{\prime \prime}$, die Gleichheit (5.1) für $j=j_{0}$ bewiesen und $j=j_{0}+1$ : Ist $k^{\prime}=k^{\prime \prime}-2^{j_{0}}$, so sind die ersten Hälften der $k^{\prime}$-ten und $k^{\prime \prime}$-ten Zeile identisch und die zweiten Hälften gehen durch Multiplikation mit -1 auseinander hervor. Daher ist

$$
\sum_{i=1}^{2 j} a_{i k^{\prime}} a_{i k^{\prime \prime}}=\sum_{i=1}^{2^{j 0}} a_{i k^{\prime}} a_{i k^{\prime}}-\sum_{i=2^{j 0}+1}^{2 j} a_{i k^{\prime}} a_{i k^{\prime}}=0 \text {. }
$$

Sei nun $k^{\prime} \neq k^{\prime \prime}-2^{j}$. Sind $k^{\prime}, k^{\prime \prime} \leqq 2^{j}$ oder $k^{\prime}, k^{\prime \prime}>2^{j}$, so ergibt $\operatorname{sich}(5.1)$ direkt aus der Induktionsannahme. Ist aber $k^{\prime} \leqq 2^{j_{0}}$ und $k^{\prime \prime}>2^{j_{0}}$, so ist $l^{\prime \prime}=k^{\prime \prime}-2^{j_{0}} \leqq 2^{j_{0}}$ und $l^{\prime \prime} \neq k^{\prime}$, ferner ist $a_{i k^{\prime \prime}}=a_{i l^{\prime \prime}}$ für $i=1, \ldots, 2^{j_{0}}$ und $a_{i k^{\prime \prime}}=-a_{i l^{\prime \prime}}$ für $i=2^{j^{+1}}, \ldots, 2^{j}$ und nun

$$
\sum_{i=1}^{2 j} a_{i k^{\prime}} a_{i k^{\prime \prime}}=\sum_{i=1}^{2^{j 0}} a_{i k^{\prime}} a_{i l^{\prime \prime}}-\sum_{i=2^{j 0+1}}^{2^{j}} a_{i k^{\prime}} a_{i l^{\prime \prime}}=0-0 .
$$

$T_{n}$ wird nun auf $L_{2}\left(m_{n}\right)$ durch $A_{n}=\left(a_{i k}\right)$ und

$$
\chi_{\left\{x_{n k}\right\}} T_{n}=2^{-\frac{1}{2} n} \sum_{i=1}^{2^{n}} a_{i k} \chi_{\left\{x_{n i}\right\}}, \quad k=1,2, \ldots, 2^{n},
$$

festgesetzt. Ist $f\left(x_{n k}\right)=b_{k}$, so schreiben wir $f \in L_{2}\left(m_{n}\right)$ in der Form $\left(b_{1}, \ldots, b_{2^{n}}\right)$. Es ist

$$
\begin{aligned}
\left\|\left(b_{1}, \ldots, b_{2^{n}}\right) T_{n}\right\|_{2^{2}} & =2^{-n}\left\|\left(\sum_{k=1}^{2^{n}} a_{1 k} b_{k}, \ldots, \sum_{k=1}^{2^{n}} a_{2^{n} k} b_{k}\right)\right\|_{2}^{2} \\
& =2^{-n} \sum_{i=1}^{2^{n}}\left(\sum_{k=1}^{2^{n}} a_{i k} b_{k}\right)^{2} \\
& =2^{-n} \sum_{i=1}^{2^{n}}\left(\sum_{k=1}^{2^{n}} b_{k}{ }^{2} a_{i k}{ }^{2}+2 \sum_{k^{\prime} \neq k^{\prime \prime}} a_{i k^{\prime}} a_{i k^{\prime \prime}} b_{k^{\prime}} b_{k^{\prime \prime}}\right) \\
& =\sum_{k=1}^{2^{n}} b_{k}{ }^{2}=\left\|\left(b_{1}, \ldots, b_{2^{n}}\right)\right\|_{2}^{2},
\end{aligned}
$$


und also

$$
\left\|T_{n}\right\|_{2}=1 \text {. }
$$

$\left|T_{n}\right|$ existiert und hat die Gestalt

Es ist

$$
\chi_{\left\{x_{n k}\right\}}\left|T_{n}\right|=2^{-\frac{1}{2} n} \sum_{i=1}^{2^{n}} \chi_{\left\{x_{n i}\right\}} .
$$

$$
\begin{aligned}
\left\|\left(b_{1}, \ldots, b_{2 n}\right)\left|T_{n}\right|\right\|_{1} & =2^{n \cdot 2^{-\frac{1}{2} n}}\left|\sum_{i=1}^{2^{n}} b_{i}\right| \\
& \leqq 2^{\frac{1}{2} n}\left\|\left(b_{1}, \ldots, b_{2^{n}}\right)\right\|_{1}
\end{aligned}
$$

mit Gleichheit, wenn die $b_{i}$ positiv sind. Ebenso gilt

$$
\begin{aligned}
\left\|\left(b_{1}, \ldots, b_{2 n}\right)|T|\right\|_{\infty} & =2^{-\frac{1}{2} n}\left|\sum_{i=1}^{2^{n}} b_{i}\right| \\
& \leqq 2^{\frac{1}{2} n}\left\|\left(b_{1}, \ldots, b_{2^{n}}\right)\right\|_{\infty}
\end{aligned}
$$

mit Gleichheit, wenn die $b_{i}$ gleich groß und positiv sind. Also ist

$$
\left\|\left|T_{n}\right|\right\|_{1}=\left\|\left|T_{n}\right|\right\|_{\infty}=2^{\frac{1}{n} n} .
$$

Nach dem Rieszschen Konvexitätssatz (Dunford-Schwartz [7, S. 525]) folgt $\left\|\left|T_{n}\right|\right\|_{2} \leqq 2^{\frac{1}{2} n}$. Es ist

$$
\left\|\left|T_{n}\right|(1, \ldots, 1)\right\|_{2}^{2}=2^{2 n}=2^{n}\|(1, \ldots, 1)\|_{2}^{2}
$$

und somit $\left\|\left|T_{n}\right|\right\|_{2}=2^{\frac{1}{2} n}$.

Bemerkung. Nimmt $f$ auf $\Omega_{n}$ den Wert $2^{-n}$ an, $n=1,2, \ldots$, so ist $f \in L_{2}\left(m^{\prime}\right)$ und also $f T \in L_{2}\left(m^{\prime}\right)$. Es ist aber in diesem Fall nicht $f|T| \in$ $L_{2}\left(m^{\prime}\right)$ und dies zeigt, daß die linearen Operatoren in $L_{2}\left(m^{\prime}\right)$ auch beim Absehen von der Stetigkeitsforderung keinen Verband bilden.

Lemma 5.1. Sei $(\Omega, B, m)$ ein Maßraum, $B_{x} \subseteq B$ ein $\sigma$-Körper, $\Omega=$ $\Omega_{x}+\Omega_{y}$ eine disjunkte $B_{x}$-meßbare Zerlegung von $\Omega$ und für $X \in B_{x}$ sei $m_{x}(X)=m\left(X \cap \Omega_{x}\right)$. Der Raum $\left(\Omega, B_{x}, m_{x}\right)$ sei $\sigma$-endlich. Bilden dann die stetigen linearen Operatoren im reellen $L_{2}(\Omega, B, m)$ einen Verband, so auch die stetigen linearen Operatoren im reellen $L_{2}\left(\Omega, B_{x}, m_{x}\right)$.

BEWEIS. Es sei $T_{x}$ ein stetiger linearer Operator in $L_{2}\left(\Omega, B_{x}, m_{x}\right)$ und $E_{x}$ die bedingte Erwartung bezüglich $B_{x}$ und $m_{x}$. Der Operator $T$ sei in $L_{2}(\Omega, B, m)$ erklärt durch

$$
f T=\left(\left(\left(f E_{x}\right) \chi_{\Omega_{x}}\right) T_{x}\right) \chi_{\Omega_{x}} .
$$

Nach Voraussetzung existiert $T^{+}=T \vee 0$. Wir setzen für $g \in L_{2}\left(\Omega, B_{x}, m_{x}\right)$

$$
g T_{x}^{(+)}=\left(\left(g \chi_{\Omega_{x}}\right) T+E_{x}\right) \chi_{\Omega_{x}} .
$$


Ist $g \geqq 0$, so ist $g T_{x}^{(+)} \geqq 0$ und

$$
\begin{aligned}
g\left(T_{x}^{(+)}-T_{x}\right) & =\left(\left(g \chi_{\Omega_{x}}\right) T+E_{x}\right) \chi_{\Omega_{x}}-\left(\left(g \chi_{\Omega_{x}}\right) T_{x}\right) \chi_{\Omega_{x}} \\
& \geqq\left(\left(g \chi_{\Omega_{x}}\right) T E_{x}\right) \chi_{\Omega_{x}}-\left(\left(g \chi_{\Omega_{x}}\right) T_{x}\right) \chi_{\Omega_{x}}=0
\end{aligned}
$$

$m_{x}$-fastüberall. Da die stetigen linearen Operatoren in $L_{2}\left(\Omega, B_{x}, m_{x}\right)$ nach Satz 2.1 einen bedingt vollständigen Verband bilden, existiert $T_{x} \vee 0$.

SATz 5.1. Die linearen stetigen Operatoren im reellen $L_{2}(\Omega, B, m)$ bilden genau dann einen Verband, wenn $L_{2}(\Omega, B, m)$ endlich-dimensional ist.

Beweis. 1. Die Bedingung ist hinreichend: Wir sagen $(\Omega, B, m)$ habe die Eigenschaft $(N)$, wenn es eine solche ganze Zahl $n \geqq 0$ gibt, daß in $\Omega$ maximal $n$ disjunkte Mengen $A_{1}, \ldots, A_{n}$ positiven endlichen Maßes existieren. Jedes $f \in L_{2}(\Omega, B, m)$ hat dann die Gestalt $f=\sum_{i=1}^{n} a_{i} \chi_{A_{i}}$, das heißt die gleiche Gestalt wie die Funktionen $f \in L_{1}$. Hat $(\Omega, B, m)$ nicht die Eigenschaft $(N)$, so gibt es in $\Omega$ abzählbar viele disjunkte Mengen $C_{k}, k=1,2, \ldots$, positiven endlichen Maßes $m_{k}$ : Ist nämlich $\left\{D_{1}, D_{2}, D_{3}\right\}$ eine disjunkte Zerlegung von $\Omega$ und hat $D_{1}$ und $D_{2}$ positives endliches Maß, so hat $(\Omega, B, m)$ unmöglich bei Einschränkung auf jedes $D_{k}$ die Eigenschaft $(N)$, also etwa auf $D_{2}$ oder $D_{3}$ nicht. Wir setzen $D_{1}=C_{1}$ und spalten vom Rest $D_{2} \cup D_{3}$ ebenso weitere $C_{k}$ ab. Insbesondere hat $L_{2}(\Omega, B, m)$ dann mindestens abzählbare Dimension.

Ist $L_{2}$ endlich-dimensional, so haben $L_{2}$ und $L_{1}=L_{2}$ äquivalente Normen. (Siehe z. B. M. A. Neumark [15, S. 66]). Die stetigen linearen Operatoren in $L_{2}$ sind dann die gleichen wie die in $L_{1}$ und bilden nach Corollar 4.1 einen Verband.

2. Die Bedingung ist notwendig: Nun sei $L_{2}(\Omega, B, m)$ unendlich-dimensional, $\Omega_{x}=\cup_{k=1}^{\infty} C_{k}, \Omega_{y}=\Omega-\Omega_{x}, B_{x}$ von den $C_{k}$ erzeugt und $m_{x}$ wie in Lemma 5.1 erklärt. Es genügt zu zeigen, daß die linearen stetigen Operatoren auf $L_{2}\left(\Omega, B_{x}, m_{x}\right)$ keinen Verband bilden, wobei wir ohne Beschränkung der Allgemeinheit $\Omega_{y}=0$ annehmen können. Die $x_{n i} \in \Omega^{\prime}$ numerieren wir durch als $C_{k}{ }^{\prime} \subseteq \Omega^{\prime}$. Der Raum $L_{2}\left(\Omega_{x}, B_{x}, m_{x}\right)$ ist isomorph zu $L_{2}\left(\Omega^{\prime}, B^{\prime}, m^{\prime}\right)$ durch den Isomorphismus $\varphi$ :

$$
\left(\sum_{k=1}^{\infty} c_{k} \chi_{C_{k}}\right) \varphi=\sum_{k=1}^{\infty} c_{k} m_{k^{\frac{1}{2}}} \chi_{C^{\prime} k}=\sum_{k=1}^{\infty} c_{k}{ }^{\prime} \chi_{C^{\prime} k} .
$$

$\operatorname{Daß} \varphi$ ein Isomorphismus ist, folgt aus

$$
\left\|\sum_{k=1}^{\infty} c_{k} \chi_{C_{k}}\right\|_{2}^{2}=\sum_{k=1}^{\infty}\left|c_{k}{ }^{2}\right| m_{k}=\sum_{k=1}^{\infty}\left|c_{k}^{\prime}\right|^{2}=\left\|\sum_{k=1}^{\infty} c_{k}{ }^{\prime} \chi_{C^{\prime} k}\right\|_{2}^{2} .
$$

Damit ist der allgemeine Fall auf das eingangs gegebene Beispiel reduziert. 


\section{Der duale Operator.}

Satz 6.1. Ist $T$ ein majorisierbarer Operator von dem (Ko,B)-Raum $X$ in den $(K o, B)$-Raum $Y$ mit der Majorante $T_{0}$, so ist der duale Operator $T_{0}{ }^{*}$ von $T_{0}$ eine Majorante von $T^{*}$. Ist insbesondere $Y$ ein $(K o, B, b v)$ Raum und sind $X^{*}$ und $Y^{*}(K o, B)$-Räume, so gilt $\left|T^{*}\right| \leqq|T|^{*}$.

Beweis. Der Raum $X^{*}$ besteht aus den stetigen linearen Operatoren von $X$ in den $(K o, B, 1, b v)$-Raum $K$ der komplexen Zahlen und nach Satz 4.2 können wir zu $h \in X^{*}|h|$ bilden. $(f, h)$ bezeichne den Wert von $h$ für $f \in X$. Aus Satz 3.2 folgt $|(f, h)| \leqq(|f|,|h|)$. Sei nun $f \in X^{+}, h \in X^{*}$, $|h| \leqq g \in X^{*+}$. Es ist

$$
\begin{aligned}
\left|\left(f, h T^{*}\right)\right|=|(f T, h)| & \leqq(|f T|,|h|) \\
& \leqq\left(f T_{0},|h|\right) \\
& =\left(f,|h| T_{0}{ }^{*}\right) \leqq\left(f, g T_{0}{ }^{*}\right) .
\end{aligned}
$$

$g T_{0}{ }^{*}$ ist also eine Majorante von $h T^{*}$, das heißt $\left|h T^{*}\right| \leqq g T_{0}{ }^{*}$. Da dies insbesondere für $h=g$ gilt, ist $T_{0}{ }^{*}$ Majorante von $T^{*}$. Ist $Y(K o, B, b v)$ Raum, so kann man $|T|$ bilden; $|T|$ ist Majorante von $T$, also $|T|^{*}$ von $T^{*}$. Ist $X^{*}(K o, B)$-Raum, so ist aufgrund von Satz 2.1 sogar $X^{*}$ ein $(K o, B, b v)$ Raum. Da $|T|^{*}$ Majorante von $T^{*}$ ist, ist $\left|T^{*}\right| \leqq|T|^{*}$.

Wir wollen wenigstens in einigen Fällen $\left|T^{*}\right|=|T|^{*}$ beweisen:

Lemma 6.1. Ist $X$ ein bedingt vollständiger Banachverband und bedeutet $\varkappa$ die natürliche Einbettung von $X$ in $X^{* *}$, so gilt für $a_{1}, a_{2} \in X$ stets $a_{1} \varkappa v a_{2} \varkappa=\left(a_{1} \vee a_{2}\right) \varkappa$.

BeweIs. Für $b \in X^{+}$erklären wir ähnlich wie Kakutani [12, S. 526], einen Operator $P_{b}$ in $X$. Für $x \in X^{+}$sei

Aus

$$
x P_{b}=\sup _{n}(n b \wedge x) \text {. }
$$

$$
\begin{aligned}
(n b \wedge x)+(n b \wedge y) & =(n b+(n b \wedge y)) \wedge(x+(n b \wedge y)) \\
& =2 n b \wedge(n b+y) \wedge(x+n b) \wedge(x+y)
\end{aligned}
$$

folgt

$$
n b \wedge(x+y) \leqq(n b \wedge x)+(n b \wedge y) \leqq 2 n b \wedge(x+y) .
$$

Aus der Monotonie der Folgen $n b \wedge x$ und $n b \wedge y$ erhalten wir damit $x P_{b}+y P_{b}=(x+y) P_{b}$ für $x, y \in X^{+}$. Der Operator $P_{b}$ ist positiv homogen auf $X^{+}$, da für $\lambda \geqq 0$ stets

$$
\begin{aligned}
n b \wedge \lambda x & \leqq \lambda\left(\left(\left[\lambda^{-1} n\right]+1\right) b \wedge x\right) \\
& \leqq(n+[\lambda]+1) b \wedge \lambda x
\end{aligned}
$$


ist. Ferner ist $0 \leqq x P_{b} \leqq x$ und daher $\left\|P_{b}\right\| \leqq 1$. Also kann $P_{b}$ zu einem stetigen linearen Operator in $X$ fortgesetzt werden.

Ist $x \wedge b=0$, so ist $n x \wedge n b=0$ und erst recht $x \wedge n b=0$. Dann ist also $x P_{b}=0$.

Sei nun $a \in X$. Die Form $a \varkappa$ ist Linearform auf $X^{*}$ und für $L \in X^{*+}$ ist nach (3.2)

Für $0 \leqq L^{\prime} \leqq L$ ist

$$
\left(L,(a \varkappa)^{+}\right)=\sup _{0 \leqq L^{\prime} \leqq L}\left(L^{\prime}, a \varkappa\right)
$$

$$
\begin{aligned}
\left(L^{\prime}, a \varkappa\right)=\left(a, L^{\prime}\right) & =\left(a^{+}-a^{-}, L^{\prime}\right) \\
& \leqq\left(a^{+}, L^{\prime}\right) \leqq\left(a^{+}, L\right) .
\end{aligned}
$$

Umgekehrt ist durch $\left(x, L^{\prime}\right)=\left(x P_{a^{+}}, L\right)$ eine stetige Linearform $L^{\prime}$ auf $X$ definiert und für $x=a$ ist

$$
\left(L^{\prime}, a \varkappa\right)=\left(a P_{a^{+}}, L\right)=\left(a^{+} P_{a^{+}}, L\right)-\left(a^{-} P_{a^{+}}, L\right)=\left(a^{+}, L\right) .
$$

Daraus folgt $\left(L,(a \varkappa)^{+}\right)=\left(a^{+}, L\right)=\left(L,\left(a^{+}\right) \varkappa\right)$, das heißt $(a \vee 0) \varkappa=a \varkappa v 0$. Durch Translation erhalten wir daraus $a_{1} \varkappa v a_{2} \varkappa=\left(a_{1} v a_{2}\right) \varkappa$ für beliebige $a_{1}, a_{2} \in X$.

Bemerkung. Nach einer Fußnote von Kakutani [13, S. 1023], hat Alaoglu dieses Lemma sogar für beliebige Banachverbände bewiesen. Alaoglu scheint den Beweis nicht veröffentlicht zu haben.

Satz 6.2. Ist $T$ ein majorisierbarer Operator von dem $(K o, B, b v)$-Raum $X$ in den $(K o, B, m K)$-Raum $Y$, sind ferner $X^{*}, Y^{*}$ und $Y^{* *}(K o, B)$ Räume (vgl. Bemerkung (2) nach diesem Satz), so ist $\left|T^{*}\right|=|T|^{*}$.

BeweIs. Für $h \in Y$ gilt $|h| \varkappa=|h \varkappa|$. Ist nämlich $h=\sum_{i=1}^{n} a_{i} h_{i}$ mit $h_{i} \in Y^{+}$und $h_{i} \wedge h_{k}=0, i \neq k$, so ist

$$
|h| \varkappa=\left(\sum_{i=1}^{n}\left|a_{i}\right| h_{i}\right) \varkappa=\sum_{i=1}^{n}\left|a_{i}\right| h_{i} \varkappa .
$$

Da aber $h_{i} \varkappa \wedge h_{k} \varkappa=0$ für $i \neq k$ ist und $Y^{* *}$ ein $(K o, B)$-Raum ist, erhalten wir für solche $h$ stets $|h| \varkappa=|h \varkappa|$. Wegen der Stetigkeit des Übergangs zum Absolutbetrag gilt es für alle $h \in Y$. Nun ist für $g \in Y^{*+}$ und $d \in X$

$$
\begin{aligned}
|(g, d T \varkappa)|=|(d T, g)| & =\left|\left(d, g T^{*}\right)\right| \\
& \leqq\left(|d|,\left|g T^{*}\right|\right) \\
& \leqq\left(|d|, g\left|T^{*}\right|\right)=\left(g,|d| \varkappa\left|T^{*}\right|^{*}\right) .
\end{aligned}
$$

Da dies für alle $g \in Y^{*+}$ gilt, ist $|d| \varkappa\left|T^{*}\right|^{*}$ Majorante von $d T \varkappa$, das heißt

$$
|d T \varkappa| \leqq|d| \varkappa\left|T^{*}\right|^{*} \quad \text { bzw. } \quad|d T| \varkappa \leqq|d| \varkappa\left|T^{*}\right|^{*} .
$$


Für $f_{i} \in X^{+}$haben wir

$$
\left|f_{i} T\right| \varkappa \leqq f_{i} \varkappa\left|T^{*}\right|^{*} \leqq f_{i} \varkappa|T|^{* *}=f_{i}|T| \varkappa .
$$

Sei nun $f \in X^{+}$und $Z(f)=\left\{f_{1}, \ldots, f_{k}\right\}$. Setzen wir (6.1) in

ein, so folgt wegen

$$
f|T| x=\left(\lim _{Z(f) \in B(f)} \sum_{i=1}^{k}\left|f_{i} T\right|\right) x
$$

$\mathrm{da} B$

$$
\left(\sum_{i=1}^{k}\left|f_{i} T\right|\right) \varkappa=\sum_{i=1}^{k}\left(\left|f_{i} T\right| \varkappa\right) \leqq \sum_{i=1}^{k} f_{i} \varkappa\left|T^{*}\right|^{*}=f \varkappa\left|T^{*}\right|^{*},
$$

$$
f|T| \varkappa \leqq f \varkappa\left|T^{*}\right|^{*} \leqq f \varkappa|T|^{* *}=f|T| \varkappa
$$

und also Gleichheit. Für $g \in Y^{*}$ ist also

$$
\begin{aligned}
\left(f, g|T|^{*}\right)=(f|T|, g)=\left(g, f \varkappa|T|^{* *}\right) & =\left(g, f \varkappa\left|T^{*}\right|^{*}\right) \\
& =\left(g\left|T^{*}\right|, f \varkappa\right)=\left(f, g\left|T^{*}\right|\right) .
\end{aligned}
$$

Hieraus folgt $|T|^{*}=\left|T^{*}\right|$.

Bemerkungen. (1) Gelingt es, in Lemma $6.1\left(\sup f_{i}\right) \varkappa=\sup \left(f_{i} \varkappa\right)$ nicht nur für endliche Suprema zu beweisen, so genügt es, wenn $Y$ in Satz 6.2 ein $(K o, B, b v)$-Raum ist.

(2) (beim Druck): Die in den Sätzen 4.1 und 4.2 untersuchten Räume von Operatoren, insbesondere Dualräume von $(K o, B)$-Räumen, sind $(K o, B)$-Räume. Ich werde das zeigen in Ein Approximationslemma in der komplexen Hülle von Banachverbänden.

\section{Vollstetige Operatoren.}

Ein linearer stetiger Operator $T$ von einem Banachraum $X$ in einen Banachraum $Y$ heißt vollstetig, wenn das Bild ET der Einheitskugel

$$
E=\{f \in X:\|f\| \leqq 1\}
$$

bedingt norm-kompakt ist. - Wir benötigen folgende Variante eines Satzes von Mazur:

Lemma 7.1. Ist $Y$ ein komplexer Banachraum und $A \subseteq Y$ bedingt kompakt, so ist

$$
H(A)=\left\{g \in Y: g=\sum_{i=1}^{k} a_{i} g_{i} ; g_{i} \in A, \sum_{i=1}^{k}\left|a_{i}\right| \leqq 1\right\}
$$

ebenfalls bedingt kompakt. 
Beweis. Die Einheitskugel $E(K)$ des Raumes $K$ der komplexen Zahlen ist kompakt, ebenso die abgeschlossene Hülle $\bar{A}$ von $A$, also auch $\bar{A} \times E(K)$. Da $(f, c) \rightarrow c f$ stetig ist, ist $\{c f: c \in E(K), f \in \bar{A}\}$ kompakt. Satz V. 2.6 in Dunford-Schwartz [7] von Mazur besagt, daß die konvexe Hülle dieser Menge bedingt kompakt ist. Sie enthält $H(A)$.

Satz 7.1. Der Absolutbetrag $|T|$ eines vollstetigen linearen stetigen Operators $T$ von einem $(K o, B, L)$-Raum $X$ in einen $(K o, B, m K)$-Raum $Y$ ist vollstetig.

Bemerkung. Im reellen Fall bedeutet dies: Die vollstetigen Operatoren von einem $(L)$-Raum $X$ in einen $(B, V, m K)$-Raum $Y$ bilden einen linearen Unterverband des Vektorverbandes aller stetigen linearen Operatoren von $X$ nach $Y$, da nach Dunford-Schwartz [7, Satz VI 5.4] die vollstetigen Operatoren einen linearen Raum bilden.

BeweIs. Der Raum $\overline{E T}$ ist kompakt. Da die Abbildung $f \rightarrow|f|$ normstetig ist, ist $A=\{|f|: f \in \overline{E T}\}$ bedingt kompakt. (Da $f \rightarrow|f|$ nicht stets schwach stetig ist, scheint sich Satz 7.1 nicht auf schwach vollstetige Operatoren übertragen zu lassen.) $H(A)$ und $H(\overline{H(A)})$ sind bedingt kompakt.

Sei $f \in X^{+}$und $\|f\| \leqq 1$. Wir zeigen $f|T| \in \overline{H(A)}$. Da für eine geeignete Folge von Zerlegungen $Z(f)$

$$
f|T|=\lim Q^{(\sim)}(Z(f), T)
$$

ist, genügt es nachzuweisen, daß $Q^{(\sim)}(Z(f), T)$ zu $H(A)$ gehört. Sei $Z(f)=\left\{f_{1}, \ldots, f_{k}\right\}$ und $\left\|f_{i}\right\|=a_{i}>0$. Wir setzen $g_{i}=a_{i}{ }^{-1} f_{i}$. Dann ist $\sum_{i=1}^{k}\left|a_{i}\right| \leqq 1$ und $\left\|g_{i}\right\| \leqq 1$ und daher

$$
Q^{(\sim)}(Z(f), T)=\sum_{i=1}^{k} a_{i}\left|g_{i} T\right| \in H(A) .
$$

Es genügt nun, für $h \in \operatorname{Tr}(X)$ mit $\|h\| \leqq 1$

$$
h|T| \in H(\overline{H(A)})
$$

nachzuweisen. Es sei $h=\sum_{i=1}^{n} c_{i} h_{i}$ mit $h_{i} \wedge h_{k}=0, i \neq k$. Ohne Beschränkung der Allgemeinheit ist $\left|c_{i}\right|>0$ und $\left\|h_{i}\right\|>0$. Setzen wir $d_{i}=\left\|h_{i}\right\|^{-1} h_{i}$, so ist $\left\|d_{i}\right\|=1, h=\sum_{j=1}^{n}\left(c_{j}\left\|h_{j}\right\|\right) d_{j}$ und

$$
\sum_{j=1}^{n}\left(\left|c_{j}\right|\left\|h_{j}\right\|\right)=\left\|\sum_{j=1}^{n}\left|c_{j}\right| h_{j}\right\|=\||h|\|=\|h\| \leqq 1 .
$$

Demzufolge ist $h|T|=\sum_{j=1}^{n}\left(c_{j}\left\|h_{j}\right\|\right) d_{j}|T| \in H(\overline{H(A)})$. 


\section{Treppenoperatoren und starke Operatortopologie.}

Wir diskutieren nun eine Klasse von besonders einfach gebauten Operatoren. $(\Omega, B, m)$ sei ein Maßraum, $X$ der komplexe $L_{1}(\Omega, B, m)$ und $Y$ sei ein $(K o, B)$-Raum. Die nun einzuführenden Treppenoperatoren besitzen einen Absolutbetrag $|T|$, der finit bestimmbar ist, während die Bestimmung von $|T|$ im allgemeinen Grenzwertbildungen oder sogar überabzählbare Suprema erfordert. Treppenoperatoren sind stets vollstetig. Wir zeigen, daß sie in dem Sinne repräsentativ sind, daß sie stark operatordicht im Raum aller stetigen linearen Operatoren $X \rightarrow Y$ liegen.

Wir nennen einen Operator $T$ von $X$ nach $Y$ Treppenoperator, wenn er die Gestalt hat

$$
f T=\sum_{i=1}^{n} \int_{\Omega_{i}} f d m h_{i},
$$

wobei die $\Omega_{i}$ disjunkte $B$-meßbare Mengen sind und $h_{i} \in \operatorname{Tr}(Y)$ gilt. $T$ ist linear und stetig mit

denn es ist

$$
\|T\|=\underset{1 \leqq i \leqq n}{\operatorname{Max}}\left\|h_{i}\right\|=M<\infty ;
$$

$$
\|f T\| \leqq \sum_{i=1}^{n}\left\|f \chi_{\Omega_{i}} T\right\| \leqq M \cdot \sum_{i=1}^{n}\left\|f \chi_{\Omega_{i}}\right\| \leqq M\|f\|,
$$

und Gleichheit gilt für $f=\chi_{\Omega_{i}}$, wenn $i$ der Index ist, für den das Maximum angenommen wird. Für Treppenoperatoren $T$ liefert die Zerlegung

$$
Z(f)=\left\{f \chi_{\Omega_{1}}, \ldots, f \chi_{\Omega_{n}}\right\}
$$

bereits $\sup Q^{(\sim)}(Z(f), T)$. Der Raum $Y$ braucht daher nicht ein $(K o, B, b v)$ Raum zu sein. Wir erhalten

$$
f|T|=\sum_{i=1}^{n} \int_{\Omega_{i}} f d m\left|h_{i}\right| .
$$

Treppenoperatoren lassen sich auch definieren, wenn $X$ ein $(L)$-Raum ist. Wir begnügen uns mit dem Hinweis, daß nach Kakutani [12, S. 532] jeder $(L)$-Raum zu einem $\operatorname{Raum} L_{1}(\Omega, B, m)$ isomorph ist.

SaTz 8.1. Die Treppenoperatoren liegen in der starken Operatortopologie dicht im Banachraum aller stetigen linearen Operatoren vom komplexen $L_{1}(\Omega, B, m)=X$ in den $(K o, B)$-Raum $Y$.

Beweis. Sei $\varepsilon>0, f_{1}, \ldots, f_{m} \in X$ und $T$ ein stetiger linearer Operator von $X$ nach $Y$. Wir müssen einen Treppenoperator $T_{\varepsilon}$ konstruieren, für den $\left\|f_{k} T-f_{k} T_{\varepsilon}\right\|<\varepsilon$ für $k=1, \ldots, m$ gilt. Wir können $\|T\|>0$ annehmen, denn $T=0$ ist Treppenoperator. 
$\mathrm{Zu}$ jedem $f_{k}$ bestimmen wir eine Treppenfunktion $f_{k}{ }^{\prime}$ mit

$$
\left\|f_{k}-f_{k}{ }^{\prime}\right\|<\frac{1}{4} \varepsilon /\|T\|, \quad k=1, \ldots, m .
$$

Für die endlich vielen $f_{k}{ }^{\prime}$ gibt es eine Darstellung $f_{k}{ }^{\prime}=\sum_{i=1}^{n} c_{k i} \chi_{\Omega_{i}}$ mit disjunkten und gemeinsamen $\Omega_{i}$ und $0<m\left(\Omega_{i}\right)<\infty$. Wir suchen zu $\chi_{\Omega_{i}} T=y_{i}$ ein $g\left(y_{i}\right) \in \operatorname{Tr}(Y)$ mit den Eigenschaften

$$
\left\|g\left(y_{i}\right)\right\| \leqq\left\|y_{i}\right\|, \quad\left\|y_{i}-g\left(y_{i}\right)\right\|<\delta(\varepsilon)=\frac{1}{4} \varepsilon\left(\max _{k} \sum_{i=1}^{n}\left|c_{k i}\right|\right)^{-1} .
$$

Um $g\left(y_{i}\right)$ zu finden, suchen wir zunächst eine Folge $g_{j}\left(y_{i}\right) \in \operatorname{Tr}(Y)$ mit $\left\|y_{i}-g_{j}\left(y_{i}\right)\right\| \rightarrow 0$. Im Beweis von Lemma 3.1 ist gezeigt, daß solch eine Folge sogar unter der Zusatzbedingung $\left|g_{j}\left(y_{i}\right)\right| \leqq\left|y_{i}\right|$ existiert. Für ein $j$ mit $\left\|y_{i}-g_{j}\left(y_{i}\right)\right\|<\delta(\varepsilon)$ setzen wir $g\left(y_{i}\right)=g_{j}\left(y_{i}\right)$ und erhalten zugleich

$$
\left\|g\left(y_{i}\right)\right\|=\left\|\left|g_{j}\left(y_{i}\right)\right|\right\| \leqq\left\|\left|y_{i}\right|\right\|=\left\|y_{i}\right\| .
$$

Sei $h_{i}=m\left(\Omega_{i}\right)^{-1} g\left(y_{i}\right)$ und $T_{s}$ der Treppenoperator

$$
f T_{\varepsilon}=\sum_{i=1}^{n} \int_{\Omega_{i}} f d m h_{i} .
$$

$T_{\varepsilon}$ bildet $\chi_{\Omega_{i}}$ auf $g\left(y_{i}\right)$ ab. Für $1 \leqq k \leqq m$ ist

$$
\left\|f_{k}^{\prime} T-f_{k}^{\prime} T_{\varepsilon}\right\| \leqq \sum_{i=1}^{n}\left|c_{k i}\right|\left\|y_{i}-g\left(y_{i}\right)\right\|<\frac{1}{4} \varepsilon .
$$

Aus $\left\|\chi_{\Omega_{i}} T\right\| \geqq\left\|g\left(y_{i}\right)\right\|=\left\|h_{i}\right\| m\left(\Omega_{i}\right)$ folgt durch Vergleich mit (8.2) die Ungleichung $\left\|T_{\varepsilon}\right\| \leqq\|T\|$. Nun ist

$$
\begin{aligned}
\left\|f_{k} T_{\varepsilon}-f_{k} T\right\| & \leqq\left\|f_{k} T_{\varepsilon}-f_{k}{ }^{\prime} T_{\varepsilon}\right\|+\left\|f_{k}{ }^{\prime} T_{\varepsilon}-f_{k}{ }^{\prime} T\right\|+\left\|f_{k}{ }^{\prime} T-f_{k} T\right\| \\
& \leqq\left\|f_{k}-f_{k}{ }^{\prime}\right\|\left\|T_{\varepsilon}\right\|+\frac{1}{4} \varepsilon+\left\|f_{k}-f_{k}{ }^{\prime}\right\|\|T\| \\
& \leqq \frac{1}{4} \varepsilon+2\|T\|\left\|f_{k}-f_{k}{ }^{\prime}\right\|<\varepsilon, \quad k=1, \ldots, m .
\end{aligned}
$$

Bemerkung. Diese Dichtigkeitsaussage gilt erst recht, wenn man in (8.1) $h_{i} \in Y$ zuläßt. Sie gilt aber in einigen Fällen sogar noch, wenn man den Zulässigkeitsbereich für $\Omega_{i}$ und $h_{i}$ einschränkt, um z. B. bei separablem $L_{1}$ und $Y$ zu zeigen, daß der Banachraum der stetigen Operatoren separabel in der starken Operatortopologie ist.

Die Abbildung $T \rightarrow|T|$ ist im allgemeinen weder in der starken noch in der schwachen Operatortopologie stetig (Chacon und Krengel [5, Abschnitt 7]). Wir müssen uns daher mit einer Art monotoner Stetigkeit begnügen: 
Lemma 8.1. Sind $T$ und $T_{n}, n=1,2, \ldots$, majorisierbare stetige lineare Operatoren von einem $(K o, B)$-Raum $X$ in einen $(K o, B, m K)$-Raum $Y$ und strebt $T_{n}$ in der starken Operatortopologie gegen $T$, so gilt für $f \in X^{+}$:

$$
\lim _{n \rightarrow \infty}\left\|\left(f\left|T_{n}\right|-f|T|\right)-\right\|=0 .
$$

Ist $X$ ein $(B, V)$-Raum und $Y$ ein $(B, V, m K)$-Raum, so gilt ferner

$$
\lim _{n \rightarrow \infty}\left\|\left(f T_{n}{ }^{+}-f T^{+}\right)^{-}\right\|=0 .
$$

BeweIs. Zu $\varepsilon>0$ und $f \in X^{+}$wählen wir ein $Z_{\varepsilon}(f)=\left\{f_{1}, \ldots, f_{k}\right\}$ mit $\left\|f|T|-Q^{(\sim)}\left(Z_{\varepsilon}(f), T\right)\right\|<\frac{1}{2} \varepsilon$. Eine Zahl $N(\varepsilon)$ wird so bestimmt, daß für $n \geqq N(\varepsilon)$ und $i=1, \ldots, k$

$$
\left\|f_{i} T-f_{i} T_{n}\right\|<\frac{1}{2} \varepsilon / k
$$

ist. Aus $|a-b| \geqq|| a|-| b||$ folgt dann

$$
\left\|Q^{(\sim)}\left(Z_{\varepsilon}(f), T_{n}\right)-Q^{(\sim)}\left(Z_{\varepsilon}(f), T\right)\right\| \leqq \sum_{i=1}^{k}\left\|\left|f_{i} T_{n}\right|-\left|f_{i} T\right|\right\|<\frac{1}{2} \varepsilon .
$$

Nun ist $\left\|Q^{(\sim)}\left(Z_{\varepsilon}(f), T_{n}\right)-f|T|\right\|<\varepsilon$. Da bei weiterer Verfeinerung von $Z(f)$ die Folge $Q^{(\sim)}\left(Z(f), T_{n}\right)$ monoton wächst, ist

$$
\left\|\left(f\left|T_{n}\right|-f|T|\right)-\right\|<\varepsilon \quad \text { für } n \geqq N(\varepsilon) .
$$

Ganz analog wird die zweite Teilaussage bewiesen.

CoRollar 8.1. Sind $T$ und $T_{n}, n=1,2, \ldots$, majorisierbare stetige lineare Operatoren von einem $(B, V)$-Raum $X$ in einen $(B, V, m K)$-Raum $Y$, gilt $T_{n} \leqq T_{n+1}, n=1,2, \ldots$, und strebt $\left\{T_{n}\right\}$ in der starken Operatortopologie gegen $T$, so auch $\left\{T_{n}{ }^{+}\right\}$gegen $T^{+}$.

BeweIs. Sei $f \in X^{+}$, dann ist $f T \geqq f T_{n}$. Es folgt

$$
Q^{r+}\left(Z(f), T_{n}\right) \leqq Q^{r+}(Z(f), T) \leqq f T^{+},
$$

also $f T_{n}{ }^{+} \leqq f T^{+}$, das heißt $\left\|\left(f T_{n}{ }^{+}-f T^{+}\right)^{+}\right\|=0$. Zusammen mit (8.5) ergibt das

$$
\left\|f T_{n}{ }^{+}-f T^{+}\right\| \rightarrow 0
$$

und das ist hinreichend für die Konvergenz der $T_{n}{ }^{+}$gegen $T^{+}$in der starken Operatortopologie.

\section{Ein individueller Ergodensatz.}

R. V. Chacon [4] hat den folgenden Satz bewiesen:

SAtz 9.1. Sei $(\Omega, B, m)$ ein $\sigma$-endlicher Maßraum, $T$ ein linearer Operator in $L_{1}(\Omega, B, m)$ mit $\|T\|_{1} \leqq 1$ und $p_{k}, k=0,1, \ldots$, eine Folge nichtnegativer 
meßbarer Funktionen mit der Eigenschaft (A): Für alle $g \in L_{1}$ mit $|g| \leqq p_{k}$ gilt

$$
|g T| \leqq p_{k+1} \cdot
$$

Dann existiert für jedes $f \in L_{1}$ fastüberall auf $\left\{\omega: 0<\sum_{k=0}^{\infty} p_{k} \leqq \infty\right\}$

$$
\lim _{n \rightarrow \infty} \frac{\sum_{k=0}^{n} f T^{k}}{\sum_{k=0}^{n} p_{k}}<\infty .
$$

Mit Hilfe des Absolutbetrages $|T|$ ist es möglich, die Existenz von Folgen $p_{k}$ mit der Eigenschaft (A) für beliebige stetige lineare Operatoren $T$ in $L_{1}$ zu garantieren (siehe Chacon und Krengel [5]):

SAtz 9.2. Ist $(\Omega, B, m)$ ein $\sigma$-endlicher Maßraum, $T$ ein linearer Operator in $L_{1}(\Omega, B, m)$ mit $\|T\|_{1} \leqq 1, f, p \in L_{1}$ und $p \geqq 0$, so existiert fastüberall auf $\left\{\omega: 0<\sum_{k=0}^{\infty} p|T|^{k} \leqq \infty\right\}$

$$
\lim _{n \rightarrow \infty} \frac{\sum_{k=0}^{n} f T^{k}}{\sum_{k=0}^{n} p|T|^{k}}<\infty .
$$

Beweis. Es sei $p_{k}=p|T|^{k}$. Ist $|g| \leqq p|T|^{k}$, so ist

$$
|g T| \leqq|g||T| \leqq p|T|^{k+1} \text {. }
$$

Für $T \geqq 0$ ist $|T|=T$, und dann macht Satz 9.2 gerade die Aussage des Satzes von Chacon-Ornstein [6] aus. Der Beweis von Satz 9.2 gelingt auch ohne den Umweg über Satz 9.1 durch Umformulierung der von Chacon [4] entwickelten Methoden, wobei sich einige Beweisteile erübrigen.

\section{Ergodische Zerlegungen.}

Die nun beschriebenen Definitionen und Sätze sind, soweit sie nicht den Begriff des Absolutbetrags eines Operators erfordern, bereits von Jacobs [10], zum Teil schon von R. V. Chacon [3] dargestellt worden: Es sei $(\Omega, B, m)$ ein $\sigma$-endlicher Maßraum und $L_{1}$ der reelle oder komplexe $L_{1}(\Omega, B, m)=L_{1}(m)$. Wir ordnen jedem Operator $T$ in $L_{1}(m)$ den zugehörigen normierten Operator $T /\|T\|=\mathfrak{I}$ zu, den trivialen Fall $T=0$ schließen wir aus. Chacon [3, Theorem 2] hat gezeigt: Ist $T \geqq 0$ und $\|T\|=1$, so gibt es eine disjunkte Zerlegung von $\Omega$ in einen konservativen Teil $C(T)$ und einen dissipativen Teil. Ist $p \in L_{1}{ }^{+}$und fastüberall $>0$, so ist $C(T)$ gerade $\left\{\omega: \sum_{k=0}^{\infty} p T^{k}=+\infty\right\}$ unabhängig von $p$. Für beliebiges $T \neq 0$ sei $C(T)=C(|\mathfrak{T}|)$.

Eine Menge $I \in B$ heiße schwach $T$-invariant, wenn für jede außerhalb von $I$ fastüberall verschwindende Funktion $f \in L_{1}$ auch $f T$ diese Eigen- 
schaft hat. Ist außerdem $I \subseteq C(T)$, so heißt $I T$-invariant. Die Begriffe der schwach $T$-invarianten Menge und der $T$-invarianten Menge sind invariant gegen den Übergang zu $\lambda T, \lambda \neq 0$, speziell zu $\mathfrak{T}$. Chacon [3, Lemma 5] hat gezeigt, daß für positives $T$ mit $\|T\|=1$ die $T$-invarianten Mengen einen $\sigma$-Körper $B_{1}$ mit der Grundmenge $C(T)$ bilden.

Lemma 10.1. Eine Menge ist genau dann (schwach) T-invariant, wenn sie (schwach) $|T|$-invariant ist. Insbesondere bilden die $T$-invarianten $M e n$ gen einen $\sigma$-Körper $B_{1}$ mit der Grundmenge $C(T)$.

BeweIs. Ist $I$ schwach $T$-invariant, $f \in L_{1}{ }^{+}$und $\{\omega: f(\omega) \neq 0\} \subseteq I$, so gilt für alle $g \in L_{1}$ mit $|g| \leqq f$ die Inclusion $\{\omega: g(\omega) \neq 0\} \cong I$ und also auch $\{\omega: g T(\omega) \neq 0\} \cong I$ und nach Satz $3.2\{\omega: f|T|(\omega) \neq 0\} \cong I$. Für beliebige $f \in L_{1}$ mit $\{\omega: f(\omega) \neq 0\} \subseteq I$ folgt die letzte Inclusion, indem man sie zuerst für Treppenfunktionen beweist und $f$ dann approximiert. $I$ ist also auch schwach $|T|$-invariant. Ist umgekehrt eine schwach $|T|$-invariante Menge $I$ gegeben und $f \in L_{1}$ eine Funktion mit $\{\omega: f(\omega) \neq 0\} \cong I$, so ist auch $\{\omega:|f|(\omega) \neq 0\} \cong I$. Daher ist

$$
\begin{aligned}
\{\omega: f T(\omega) \neq 0\} & \cong\{\omega:|f T|(\omega) \neq 0\} \\
& \cong\{\omega:|f||T|(\omega) \neq 0\} \cong I .
\end{aligned}
$$

Da $C(T)=C(|T|)$ ist, sind auch die $T$-invarianten Mengen gerade die $|T|$-invarianten Mengen.

$(T, m)$ heißt ergodisch, wenn eine disjunkte Zerlegung $C(T)=E \cup F$ von $C(T)$ in invariante Mengen $E, F$ positiven Maßes unmöglich ist.

Ziel ist es, stetige lineare Operatoren $T \neq 0$ in $L_{1}$ in einem zu definierenden Sinn in ergodische $T_{\omega}$ zu zerlegen.

Sei $K(B)$ der in Abschnitt 1, Beispiel 2 beschriebene $(K o, B)$-Raum der komplexen Ladungsverteilungen auf $(\Omega, B)$. Ist $B_{0} \leqq B$ ein weiterer $\sigma$-Körper, so sei $m_{0}$ die Einschränkung von $m$ auf $B_{0}$. Wir betrachten Scharen $\hat{p}=\left\{p_{\omega}, \omega \in \Omega\right\}$ mit Werten $p_{\omega} \in K(B)$. Eine Schar $\hat{p}$ heißt $B_{0^{-}}$ meßbar, wenn für jedes $F \in B p_{\omega}(F)$ eine $B_{0}$-meßbare Funktion ist, und $\hat{p}$ heißt $m_{0}$-integrabel, wenn

$$
\|\hat{p}\|=\int_{\Omega}\left\|p_{\omega}\right\| m_{0}(d \omega)<\infty
$$

ist. Zwei Scharen $\hat{p}$ und $\hat{q}$ heißen $m_{0}$-äquivalent, wenn $m_{0}$-fastüberall $p_{\omega}=q_{\omega}$ ist, d. h. wenn $\|\hat{p}-\hat{q}\|=0$ ist. Wir identifizieren die Elemente der dadurch festgelegten Äquivalenzklassen miteinander. Die Schar $\hat{p}$ heiße totalstetig bzgl. $\hat{q}$, in Zeichen $\hat{p} \ll \hat{q}$, wenn $p_{\omega} \ll q_{\omega}$ für $m_{0}$-fastalle $\omega$ gilt. Es sei 
ferner

$$
L_{1}(\hat{m})=\{\hat{p}:\|\hat{p}\|<\infty \text { und } \hat{p} \gg \hat{m}\}
$$

$$
L_{\mathbf{1}}{ }^{\prime}(m)=\{p \in K(B): p \ll m\} .
$$

Den durch den Satz von Radon-Nikodym gegebenen Isomorphismus von $L_{1}(m) \rightarrow L_{1}{ }^{\prime}(m)$ bezeichnen wir mit $\psi$.

Ist $q \in K(B)$, so heiße eine $B_{0}$-meßbare Schar $\hat{q}=\left\{q_{\omega}: \omega \in \Omega\right\}$ eine Zerlegung von $q$ nach $B_{0}$, wenn

$$
q(F \cap G)=\int_{G} q_{\omega}(F) m_{0}(d \omega) \quad \text { für } \quad F \in B, G \in B_{0}
$$

gilt. Ist $m(\Omega)=1$, so ist $\hat{m}$ gerade eine bedingte Wahrscheinlichkeitsverteilung von $m$ bzgl. $B_{0}$. Existiert zu jedem $B_{0} \subseteq B$ eine Zerlegung $\hat{m}$, so sagen wir, $(\Omega, B, m)$ habe die Eigenschaft $(\hat{B})$. (Siehe z. B. Jacobs $[9$, Kap. $9, \S 6]$.)

Im folgenden sei $B$ abzählbar erzeugt. Von Jacobs [10] übernehmen wir folgende Tatsachen in leichter Verallgemeinerung aufs Komplexe: Existiert eine Zerlegung $\hat{m}$ von $m$ nach $B_{0}$, so besitzt jedes $p \in L_{\mathbf{1}}{ }^{\prime}(m)$ eine Zerlegung $\hat{p}$ nach $B_{0}$. Ist $d p=f d m$, so ist

$$
d p_{\omega}=f d m_{\omega}
$$

für $m_{0}$-fastalle $\omega$. Bezeichnet $K(\hat{m})$ die Menge aller $\hat{p} \in L_{1}(\hat{m})$, die gemä $\beta$ (10.2) durch Funktionen $f \in L_{1}(m)$ gegeben sind, so ist die Abbildung $\varphi: p \rightarrow \hat{p}$ ein Isomorphismus von $L_{1}^{\prime}(m)$ und $K(\hat{m})$.

Der Beweis beruht auf folgender Tatsache: Für $f \in L_{1}(m)$ und $G \in B_{0}$ ist

$$
\int_{G} f d m=\int_{G}\left(\int_{\Omega} f d m_{\omega}\right) m_{0}(d \omega) .
$$

Wir merken uns, daß ein in der Gestalt (10.2) gegebenes $\left\{p_{\omega}\right\} B_{0}$-meßbar ist und, daß jedes $f \in L_{1}(m)$ für $m_{0}$-fastalle $\omega$ zu $L_{1}\left(m_{\omega}\right)$ gehört.

Sei nun $T$ ein stetiger linearer Operator in $L_{1}(m) . L_{1}(m)$ ist durch $f \rightarrow f \psi \varphi$ isomorph mit $K(\hat{m})$ und der Isomorphismus $\varrho=\psi \varphi$ induziert einen stetigen linearen Operator $\hat{T}=\varrho^{-1} T \varrho$ in $K(\hat{m})$. Da auch $\hat{T}$ umgekehrt $T$ bestimmt, heißt $\hat{T}$ die zu $m$ gehörige Darstellung von $T$.

Eine Schar $\left\{T_{\omega}, \omega \in \Omega\right\}$ von stetigen linearen Operatoren $T_{\omega}$ in $L_{1}{ }^{\prime}\left(m_{\omega}\right)$ heißt eine zu $\hat{m}$ gehörige Zerlegung von $T$, wenn:

(a) $\left(p_{\omega} T_{\omega}\right)(F)$ für jedes $F \in B$ und jedes $\hat{p} \in K(\hat{m})$ eine $B_{0}$-meßbare

Funktion ist, und

(b) für jedes $\hat{p} \in(\hat{m})$ die Gleichung

$$
(\hat{p} \hat{T})_{\omega}=p_{\omega} T_{\omega}
$$

$m_{0}$-fastüberall gilt. 
Die Forderung (b) ist sinnvoll, weil $p_{\omega}$ für $m_{0}$-fastalle $\omega$ zu $L_{1}{ }^{\prime}\left(m_{\omega}\right)$ gehört. Jacobs [10] hat gezeigt, daß bei gegebenem $T$ und $\hat{m}$ die Schar $\left\{T_{\omega}\right\} m_{0}$-fasteindeutig durch (a) und (b) bestimmt ist. Der folgende Satz garantiert unter einschränkenden Bedingungen die Existenz:

SATz 10.1. Sei $(\Omega, B, m)$ ein $\sigma$-endlicher Maßraum, $B$ abzählbar erzeugt und $B_{0} \subseteq B$, ferner $\hat{m}$ eine Zerlegung von $m$ nach $B_{0}$. Ist dann $T$ ein stetiger linearer Operator in $L_{1}(m)$ und sind alle Mengen $G \in B_{0}$ schwach $T$-invariant, so existiert eine zu $\hat{m}$ gehörige Zerlegung $\hat{T}=\left\{T_{\omega}: \omega \in \Omega\right\}$ mit $\left\|T_{\omega}\right\| \leqq\|T\|$.

Beweis. Sei $R$ die Menge der komplexen Zahlen mit rationalem Realund Imaginärteil, $K_{0}$ ein abzählbarer $B$ erzeugender Mengenkörper, dessen Mengen $X$ endliches Maß $m(X)$ haben, und $L_{R}=\left\{f=\sum_{j=1}^{n} a_{i} \chi_{A_{i}}, a_{i} \in R\right.$, $\left.A_{i} \in K_{0}\right\}$. Der Raum $L_{R}$ ist dann ein in $L_{1}(m)$ dichter linearer Raum mit Skalarbereich $R$ und er enthält nur abzählbar viele Elemente. Für $m_{0}$-fastalle $\omega$ haben alle $X \in K_{0}$ endliches $\mathrm{Maß} m_{\omega}(X)$ und für diese liegt $L_{R}$ auch dicht in $L_{1}\left(m_{\omega}\right)$.

Zu $f \in L_{R}$ suchen wir $\hat{p}=f \varrho$, sodaß $m_{0}$-fastüberall $d p_{\omega}=f d m_{\omega}$ gilt. Ist dann $\hat{q}=\hat{p} \hat{T}$, so ist $\hat{q}=f \varrho \varrho^{-1} T \varrho=f T \varrho$ und also $d q_{\omega}=(f T) d m_{\omega} m_{0}$-fastüberall. Da nur abzählbar viele $\hat{p}$ und $\hat{q}$ so gebildet sind, können wir durch Elimination einer Nullmenge $N \in B_{0}$ dafür sorgen, daß für $\omega \notin N$

$$
d p_{\omega}=f d m_{\omega} \quad \text { und } \quad d q_{\omega}=(f T) d m_{\omega}
$$

gilt. Die Abbildungen $T_{\omega}: p_{\omega} \rightarrow q_{\omega}$ sind dann für $\omega \notin N$ linear mit dem Skalarbereich $R$. Da $\hat{m} B_{0}$-meßbar ist, ist $\hat{q} B_{0}$-meßbar. Ist nun $G \in B_{0}$, so sind nach Lemma $10.1 G$ und $\Omega-G$ schwach- $|T|$-invariant. Anwendung der Formel (10.3) ergibt dann:

$$
\begin{aligned}
\int_{G}\left\|q_{\omega}\right\| m_{0}(d \omega) & =\int_{G}\left(\int_{\Omega}|f T| d m_{\omega}\right) m_{0}(d \omega) \\
& =\int_{G}|f T| d m \\
& \leqq \int_{G}|f||T| d m \\
& =\int_{G}\left(|f| \chi_{G}\right)|T| d m+\int_{G}\left(|f| \chi_{\Omega-G}\right)|T| d m \\
& \leqq \int_{\Omega}\left(\chi_{G}|f|\right)|T| d m+0 \leqq\left\|T\left|\| \int_{G}\right| f \mid d m\right. \\
& =\|T\| \int_{G}\left(\int_{\Omega}|f| d m_{\omega}\right) m_{0}(d \omega)=\|T\| \int_{G}\left\|p_{\omega}\right\| m_{0}(d \omega) .
\end{aligned}
$$


Außerhalb einer $m_{0}$-Nullmenge $N_{1} \supseteqq N$ ist nun $\left\|q_{\omega}\right\| \leqq\|T\|\left\|p_{\omega}\right\|$ und daher sind die $T_{\omega}$ normstetig mit der Schranke $\left\|T_{\omega}\right\| \leqq\|T\|$. Durch stetige Fortsetzung erhält man $T_{\omega}$ als stetige lineare Operatoren in $L_{1}{ }^{\prime}\left(m_{\omega}\right)$ für $\omega \notin N_{1}$. Für $\omega \in N_{1}$ sei zum Beispiel $p_{\omega} T_{\omega}=0, p_{\omega} \in L_{1}{ }^{\prime}\left(m_{\omega}\right)$.

(10.4) ist für $\omega \notin N_{1}$ und $\hat{p} \in L_{R} \varrho$ nach Konstruktion erfüllt. Sei nun $f \in L_{1}(m)$ und $\hat{p}=f \varrho=\left\{p_{\omega}\right\}$ fest, also außerhalb einer $m_{0}$-Nullmenge $d p_{\omega}=f d m_{\omega}$, und sei $f_{k} \in L_{R}$ eine Folge mit $\left\|f_{k}-f\right\| \rightarrow 0$, ferner $\hat{p}_{k}=\left\{p_{k \omega}\right\}=f_{k} \varrho$. Wegen der Stetigkeit der $T_{\omega}$ gilt dann

$$
\begin{aligned}
\int_{\Omega}\left\|p_{\omega} T_{\omega}-p_{k \omega} T_{\omega}\right\| m_{0}(d \omega) & \leqq\|T\| \int_{\Omega}\left\|p_{\omega}-p_{k \omega}\right\| m_{0}(d \omega) \\
& =\|T\|\left\|f-f_{k}\right\| \rightarrow 0 .
\end{aligned}
$$

Andererseits gilt

$$
\int_{\Omega}\left\|(\hat{p} \hat{T})_{\omega}-p_{k \omega} T_{\omega}\right\| m_{0}(d \omega)=\int_{\Omega}\left(\int_{\Omega}\left|f T-f_{k} T\right| d m_{\omega}\right) m_{0}(d \omega) \rightarrow 0,
$$

da für die $f_{k}(10.4)$ bereits gilt. Es folgt, daß $m_{0}$-fastüberall $p_{\omega} T_{\omega}=(\hat{p} \hat{T})_{\omega}$ ist.

Jacobs [10] hat Satz 10.1 bereits für den reellen $L_{1}(m)$ und $T \geqq 0$ bewiesen.

Sei $\psi_{\omega}$ der Isomorphismus $L_{1}\left(m_{\omega}\right) \rightarrow L_{1}{ }^{\prime}\left(m_{\omega}\right)$. Die Operatoren $\psi_{\omega} T_{\omega} \psi_{\omega}{ }^{-1}$, $\psi_{\omega}\left|T_{\omega}\right| \psi_{\omega}{ }^{-1}$ in $L_{1}\left(m_{\omega}\right)$ identifizieren wir mit $T_{\omega},\left|T_{\omega}\right|$ usw.

Lemma 10.2. Unter den Voraussetzungen von Satz 10.1 seien $\hat{T}=\left\{T_{\omega}\right\}$ und $|\hat{T}|=\left\{|T|_{\omega}\right\}$ Zerlegungen von $T$ und $|T|$. Dann gilt für $m_{0}$-fastalle $\omega \in \Omega$

d.h. es ist

$$
|T|_{\omega}=\left|T_{\omega}\right|
$$

$$
f\left|T_{\omega}\right|=f|T|_{\omega} \quad \text { für } f \in L_{1}\left(m_{\omega}\right) .
$$

BEwEIs. Abgesehen von einer $m_{0}$-Nullmenge $N$ gilt für alle $\omega$, daß $L_{R} \cong$ $L_{1}\left(m_{\omega}\right)$ und darin dicht. Aufgrund der Stetigkeit und Linearität der $\left|T_{\omega}\right|$ und $|T|_{\omega}$ genügt es also (10.5) für $f \in L_{R}{ }^{+}$zu zeigen. Außerhalb einer Nullmenge $N_{2} \supseteqq N$ ist $f T_{\omega}=f T$ und $f|T|_{\omega}=f|T|$. Es ist

$$
f\left|T_{\omega}\right|=\lim Q^{(\sim)}\left(Z(f), T_{\omega}\right) .
$$

Da $L_{R}{ }^{+}$dicht in $L_{1}{ }^{+}\left(m_{\omega}\right)$ liegt, beschränken wir uns auf Zerlegungen $Z(f)=\left\{f_{1}, \ldots, f_{k}\right\}$ mit $f_{i} \in L_{R^{+}}$. Für sie ist außerhalb von $N_{2}$

$$
Q^{(\sim)}\left(Z(f), T_{\omega}\right)=\sum_{i=1}^{k}\left|f_{i} T_{\omega}\right|=\sum_{i=1}^{k}\left|f_{i} T\right|=Q^{(\sim)}(Z(f), T)
$$

und daher $f\left|T_{\omega}\right|=f|T|=f|T|_{\omega}$. 
Wir nennen nun eine Zerlegung $T=\left\{T_{\omega}\right\}$ ergodisch, wenn $m_{0}$-fastalle $\left(T_{\omega}, m_{\omega}\right)$ ergodisch sind.

SATz 10.2. Sei $(\Omega, B, m)$ ein $\sigma$-endlicher Maßraum mit der Eigenschaft $(\hat{B})$ und $B$ abzählbar erzeugt, $T \neq 0$ sei ein linearer stetiger Operator in $L_{1}(m)$ und $C(T)=\Omega$. Sei $B_{0}=B_{1}$ der $\sigma$-Körper der $T$-invarianten Mengen und $\hat{m}$ eine Zerlegung von $m$ bzgl. $B_{0}$. Der Raum $\left(\Omega, B_{0}, m\right)$ sei $\sigma$-endlich.

Die $m_{0}$-fasteindeutig bestimmte zu $\hat{m}$ gehörige Zerlegung $\hat{T}=\left\{T_{\omega}\right\}$ von $T$ ist dann ergodisch.

Bewers. Satz 10.2 ist für den reellen Fall und positives $T$ mit $\|T\|=1$ und endliches Maß $m$ von Jacobs [10] bewiesen worden, der auch gezeigt hat, wie der Fall von $\sigma$-endlichem Maß durch Isomorphieüberlegungen auf den Fall endlichen Maßes $m$ reduzierbar ist. Im komplexen Fall ist der Beweis fast wörtlich übertragbar. Die Bedingung $\|T\|=1$ erweist sich durch die abgeänderte Definition von $C(T)$ als unwesentlich: Ist $\|T\| \neq 1$, so bildet man $\mathfrak{I}=T /\|T\| . \operatorname{Da}\left\{T_{\omega}\right\}=\left\{\|T\| \mathfrak{I}_{\omega}\right\}$ ist, ist $\left\{T_{\omega}\right\}$ genau dann ergodisch, wenn $\left\{\mathfrak{I}_{\omega}\right\}$ ergodisch ist. Die Positivitätsannahme für $T$ schließlich wird durch Lemma 10.2 überflüssig, denn aus der Ergodizität von $\left\{|T|_{\omega}\right\}$ folgt die von $\left\{T_{\omega}\right\}$, da $m_{0}$-fastüberall $|T|_{\omega}=$ $\left|T_{\omega}\right|$ ist und $T_{\omega}$ nach Lemma 10.1 die gleichen invarianten Mengen besitzt wie $\left|T_{\omega}\right|$.

\section{LITERATUR}

1. G. Birkhoff, Lattice theory (Amer. Math. Soc. Colloquium Publ. 25), New York, 1948.

2. N. Bourbaki, Integration IV, (Act. Sci. Ind. 1175) Paris, 1952.

3. R. V. Chacon, Identification of the limit of operator averages, J. Math. Mech. 11 (1962), 961-968.

4. R. V. Chacon, Convergence of operator averages, erscheint noch.

5. R. V. Chacon and U. Krengel, Linear modulus of a linear operator, Proc. Amer. Math. Soc., erscheint noch.

6. R. V. Chacon and D. S. Ornstein : A general ergodic theorem, Illinois J. Math. 4 (1960), 153-160.

7. N. Dunford and J. T. Schwartz, Linear operators I, General theory, New York, 1958.

8. N. Dunford and J.T. Schwartz, Convergence almost everywhere of operator-averages, J. Math. Mech. 5 (1956), 129-178.

9. K. Jacobs, Neuere Methoden und Ergebnisse der Ergodentheorie (Ergebn. Math. Grenzgebiete, Neue Folge 29), Berlin · Göttingen · Heidelberg, 1960.

10. K. Jacobs, Ergodische Zerlegungen positiver Kontraktionen, unveröffentlichtes Manuskript, enthalten in:

11. K. Jacobs, Lectures on ergodic theory, Vorlesungsausarbeitung, Aarhus, 1963.

12. S. Kakutani, Concrete representation of abstract $(L)$-spaces and the mean ergodic theorem, Ann. of Math. (2) 42 (1941), 523-537. 
13. S. Kakutani, Concrete representation of abstract (M)-spaces, Ann. of Math. (2) 42 (1941), 994-1023.

14. L. Kantorovitch, Linear operations in semi-ordered spaces, Mat. Sbornik 49 (1940), 209-284.

15. M. A. Neumark, Normierte Algebren, Berlin, 1959.

16. F. Riesz, Sur quelques notions fondamentales dans la théorie générale des opérations linéaires, Ann. of Math. (2) 41 (1940), 174-206.

UNIVERSITÄT GÖTTINGEN, DEUTSCHLAND

UND

UNIVERSITÄT AARHUS, DÄNEMARK 